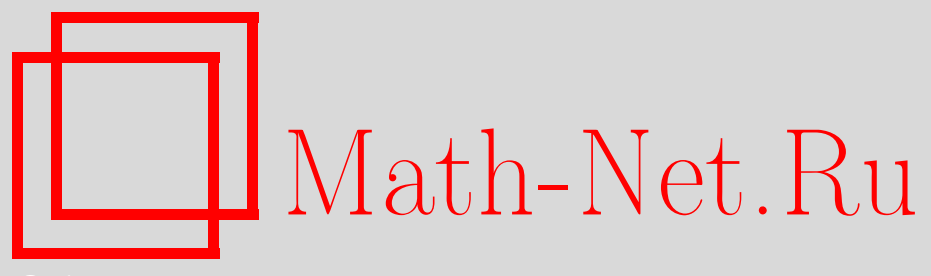

В. В. Горяйнов, О. С. Кудрявцева, Однопараметрические полугруппы аналитических функций, неподвижные точки и функция Кёнигса, Матем. сб., 2011, том 202, номер 7, 43-74

DOI: https://doi.org/10.4213/sm7708

Использование Общероссийского математического портала Math-Net.Ru подразумевает, что вы прочитали и согласны с пользовательским соглашением http://www . mathnet.ru/rus/agreement

Параметры загрузки:

IP : 18.207 .199 .55

26 апреля 2023 г., 18:22:33

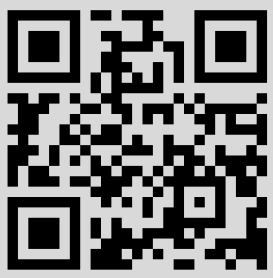




\author{
В. В. Горяйнов, О. С. Кудрявцева
}

\title{
Однопараметрические полугруппы аналитических функций, неподвижные точки и функция Кёнигса
}

\begin{abstract}
Получены аналоги формулы Берксона-Порты инфинитезимальной образующей однопараметрической полугруппы голоморфных отображений единичного круга в себя для случая, когда кроме точки Данжуа-Вольфа имеются другие неподвижные точки. С каждой однопараметрической полугруппой ассоциируется так называемая функция Кёнигса, которая является общим решением для всех элементов однопараметрической полугруппы некоторого функционального уравнения (Шрёдера - в случае внутренней точки Данжуа-Вольфа и Абеля - в случае граничной точки Данжуа-Вольфа). Дано параметрическое представление классов функций Кёнигса как с учетом точки Данжуа-Вольфа, так и с учетом других неподвижных точек отображений однопараметрической полугруппы.
\end{abstract}

Библиография: 19 названий.

Ключевые слова: однопараметрическая полугруппа, инфинитезимальная образующая, неподвижные точки, дробные итерации, функция Кёнигса.

\section{§ 1. Введение}

Пусть $\mathfrak{P}$ - совокупность всех голоморфных в единичном круге $\mathbb{D}=\{z \in \mathbb{C}$ : $|z|<1\}$ функций $f$, принимающих значения из $\mathbb{D}$. Тогда $\mathfrak{P}$ можно рассматривать как топологическую полугруппу относительно операции композиции и топологии локально равномерной сходимости. Роль единицы в этой полугруппе играет тождественное преобразование $f(z) \equiv z$. Рассматривая $\mathbb{R}^{+}=\{t \in \mathbb{R}$ : $t \geqslant 0\}$ как аддитивную полугруппу с обычной топологией вещественных чисел, под однопараметрической полугруппой в $\mathfrak{P}$ будем понимать непрерывный гомоморфизм $t \mapsto f^{t}$, действующий из $\mathbb{R}^{+}$в $\mathfrak{P}$. Это означает, что семейство $\left\{f^{t}\right\}_{t \geqslant 0}$ функций из $\mathfrak{P}$ удовлетворяет условиям:

(i) $f^{t+s}(z)=f^{t} \circ f^{s}(z)$ при $s, t \geqslant 0$;

(ii) $f^{t}(z) \rightarrow z$ локально равномерно в $\mathbb{D}$ при $t \rightarrow 0$.

Заметим, что при целых неотрицательных значениях $t$ мы получаем обычные итерации $f^{n}=f \circ f^{n-1}, n=2,3, \ldots$, функции $f=f^{1}$. Поэтому элементы семейства $\left\{f^{t}\right\}_{t \geqslant 0}$ называют также дробными итерациями функции $f$. Исследование проблемы дробных итераций имеет богатую историю и восходит к работам Шрёдера [1] и Кёнигса [2] конца 19-го века. Отметим также, что однопараметрические полугруппы аналитических функций возникают во многих вопросах анализа и его приложений. Часто они описывают динамику того или иного процесса. 
Синтез алгебраических и топологических свойств приводит к тому, что семейство $\left\{f^{t}\right\}_{t \geqslant 0}$ является дифференцируемым по $t$ (см. [3]). Более того, оно даже бесконечно дифференцируемо (см., например, [4]). Производная

$$
\left.\frac{\partial}{\partial t} f^{t}(z)\right|_{t=0}=\lim _{t \rightarrow 0} \frac{f^{t}(z)-z}{t}=v(z)
$$

представляет собой аналитическую в $\mathbb{D}$ функцию и вполне характеризует однопараметрическую полугруппу $t \mapsto f^{t}$ посредством дифференциального уравнения

$$
\frac{\partial}{\partial t} f^{t}(z)=v\left(f^{t}(z)\right)
$$

и начального условия $\left.f^{t}(z)\right|_{t=0}=z$. Функцию $v$ называют инфинитезимальной образующей однопараметрической полугруппы $t \mapsto f^{t}$. В случае, когда функции $f^{t}$ однопараметрической полугруппы $t \mapsto f^{t}$ в $\mathfrak{P}$ оставляют неподвижным начало координат и сохраняют положительное направление вещественной оси в нуле, вид инфинитезимальной образующей $v$ был получен в знаменитой работе Лёвнера [5]. В общем случае функция $f \in \mathfrak{P}$ может не иметь в круге $\mathbb{D}$ неподвижных точек. Однако классический результат, известный как теорема Данжуа-Вольфа (см., например, [6]), утверждает, что если $f \in \mathfrak{P}$ отлична от дробно-линейного преобразования единичного круга на себя, то существует единственная точка $q$ из замыкания $\overline{\mathbb{D}}$ единичного круга $\mathbb{D}$ такая, что $f^{n}(z) \rightarrow q$ локально равномерно в $\mathbb{D}$ при $n \rightarrow \infty$. При этом если $q \in \mathbb{D}$, то $f(q)=q$, т.е. $q$ является неподвижной точкой отображения $f: \mathbb{D} \rightarrow \mathbb{D}$. Если $q$ является граничной точкой, т.е. лежит на единичной окружности $\mathbb{T}=\{z \in \mathbb{C}:|z|=1\}$, то в этой точке существуют угловые пределы

$$
f(q)=\lim _{z \rightarrow q} f(z), \quad f^{\prime}(q)=\lim _{z \rightarrow q} f^{\prime}(z)=\lim _{z \rightarrow q} \frac{f(z)-q}{z-q} .
$$

Кроме того, $f(q)=q$ и $0<f^{\prime}(q) \leqslant 1$, т.е. $q$ является граничной неподвижной притягивающей точкой. В литературе $q$ называют точкой Данжуа-Вольфа функции $f$, и она является общей для всех итераций этой функции. Таким образом, если $t \mapsto f^{t}$ - однопараметрическая полугруппа в $\mathfrak{P}$, то все функции $f^{t}, t>0$, имеют одну и ту же точку Данжуа-Вольфа $q$. С учетом этого записывается (см. [3]) вид инфинитезимальной образующей:

$$
v(z)=(q-z)(1-\bar{q} z) p(z),
$$

где $p$ - голоморфная в $\mathbb{D}$ функция, удовлетворяющая условию $\operatorname{Re} p(z) \geqslant 0$ при $z \in \mathbb{D}$. Обратно, всякая функция $v$ вида (1) является инфинитезимальной образующей некоторой однопараметрической полугруппы $t \mapsto f^{t}$ в $\mathfrak{P}$ с точкой Данжуа-Вольфа q. Равенство (1) известно также как формула Берксона-Порты и лежит в основе многих исследований.

Целью настоящей работы является получение аналога формулы (1) с учетом других неподвижных точек функций однопараметрической полугруппы. Другое направление наших исследований связано с асимптотическим поведением однопараметрических полугрупп и их описанием посредством функциональных уравнений. 
Пусть $f \in \mathfrak{P}$ отлична от дробно-линейного преобразования единичного круга на себя и имеет точку Данжуа-Вольфа $q \in \mathbb{D}$. Если производная в этой точке $f^{\prime}(q)=\gamma$ не обращается в нуль, то (см., например, [6; гл. VI]) существует предел

$$
\lim _{n \rightarrow \infty} \frac{f^{n}(z)-q}{\gamma^{n}}=F(z),
$$

который представляет собой голоморфную в $\mathbb{D}$ функцию, удовлетворяющую условиям $F(q)=0, F^{\prime}(q)=1$. Это и есть функция Кёнигса, которая также является решением функционального уравнения Шрёдера

$$
F(f(z))=\gamma F(z)
$$

В вероятностных приложениях (см., например, [7]) функция Кёнигса как предел нормированной последовательности итераций связана с предельными теоремами и распределениями.

Если $t \mapsto f^{t}$ - однопараметрическая полугруппа в $\mathfrak{P}$ с точкой Данжуа-Вольфа $q \in \mathbb{D}$, то в силу однолистности функций $f^{t}, t \geqslant 0$ (это следует из единственности решения задачи Коши для обыкновенного дифференциального уравнения, воспроизводящего однопараметрическую полугруппу по инфинитезимальной образующей), производная $\left(f^{t}\right)^{\prime}(q)=\gamma^{t}$ в нуль не обращается. Поэтому можно определить функцию Кёнигса $F$, которая будет общей для всех $f^{t}, t>0$. При этом $F$ будет однолистной и

$$
F\left(f^{t}(z)\right)=\gamma^{t} F(z)
$$

т.е. функцию Кёнигса $F$ можно использовать для получения итераций функции $f$ посредством функционального уравнения Шрёдера.

В случае граничной точки Данжуа-Вольфа, т.е. $q \in \mathbb{T}$, нет полного аналога определения функции Кёнигса как предела некоторой нормированной последовательности итераций. Однако для однопараметрической полугруппы $t \mapsto f^{t}$ в $\mathfrak{P}$ с граничной точкой Данжуа-Вольфа естественно возникает функция $F$, которая удовлетворяет уравнению Абеля

$$
F\left(f^{t}(z)\right)=F(z)+t
$$

где $t \geqslant 0$, и также может быть использована для получения дробных итераций.

В настоящей работе получено параметрическое представление классов функций Кёнигса, отвечающих однопараметрическим полугруппам в $\mathfrak{P}$ с учетом наличия неподвижных точек.

Отметим также, что связь между наличием неподвижных точек у однопараметрической полугруппы и поведением функции Кёнигса изучалась в работе [8]. Кроме того, аналог формулы Берксона-Порты в случае граничной точки был получен в [9]. Результаты, приведенные ниже, позволяют конструктивно получать функцию Кёнигса и однопараметрические полугруппы с заданными неподвижными точками.

В $§ 2$ приводится терминология и формулируются основные результаты, касающиеся случая, когда наряду с точкой Данжуа-Вольфа имеется еще одна неподвижная точка у элементов однопараметрической полугруппы. Некоторым вспомогательным результатам, которые понадобятся для доказательства 
основных теорем и позволят расширить их формулировки на случай нескольких неподвижных точек, посвящен $\S 3$. В $\S 4$ содержится доказательство теоремы 1 - аналога формулы Берксона-Порты в случае одной дополнительной неподвижной точки, кроме точки Данжуа-Вольфа. Описанию классов функций Кёнигса однопараметрических полугрупп с внутренней и граничной точками Данжуа-Вольфа посвящены $\S 5$ и $\S 6$ соответственно. При этом изучен вопрос, как меняется вид функции Кёнигса, если кроме точки Данжуа-Вольфа однопараметрическая полугруппа имеет еще одну неподвижную точку. В $§ 7$ содержится обобщение формулы Берксона-Порты для нескольких неподвижных точек. Здесь также получены параметрические представления классов функций Кёнигса, позволяющих получать однопараметрические полугруппы с заданным множеством неподвижных точек.

\section{§ 2. Терминология и формулировка основных результатов}

Обозначим через $\mathfrak{P}[q], q \in \overline{\mathbb{D}}$, совокупность функций $f$ из $\mathfrak{P}$, для которых $q$ является точкой Данжуа-Вольфа. Очевидно, что всякая однопараметрическая полугруппа $t \mapsto f^{t}$ в $\mathfrak{P}$, у которой $f^{t}, t>0$, не являются дробно-линейными преобразованиями единичного круга на себя, представляет собой однопараметрическую полугруппу в $\mathfrak{P}[q]$ при некотором $q \in \overline{\mathbb{D}}$. Таким образом, формула (1) определяет вид инфинитезимальных образующих однопараметрических полугрупп в $\mathfrak{P}[q]$.

Если $f \in \mathfrak{P}$ отлична от тождественного преобразования, то в силу леммы Шварца внутри единичного круга $\mathbb{D}$ других неподвижных точек, кроме точки Данжуа-Вольфа, функция $f$ иметь не может. С другой стороны, у нее могут быть неподвижные (в смысле углового предела) точки на границе $\mathbb{T}$ единичного круга. При этом если $t \mapsto f^{t}$ - однопараметрическая полугруппа в $\mathfrak{P}$, то для всех $f^{t}, t>0$, множество неподвижных точек должно быть одним и тем же (см., например, [8]).

Для $q \in \overline{\mathbb{D}}$ и $a \in \mathbb{T}, a \neq q$, через $\mathfrak{P}[q ; a]$ обозначим подмножество функций $f$ из $\mathfrak{P}$, для которых $q$ является точкой Данжуа-Вольфа и в точке $a$ существуют угловые пределы

$$
\lim _{z \rightarrow a} f(z)=a, \quad \lim _{z \rightarrow a} f^{\prime}(z)=f^{\prime}(a)<\infty .
$$

Другими словами, $\mathfrak{P}[q ; a]$ является подполугруппой полугруппы $\mathfrak{P}[q]$, функции $f$ которой оставляют неподвижной точку $a$ и имеют в ней конечные угловые производные.

Следующий результат можно рассматривать как аналог формулы Берксона-Порты для полугруппы $\mathfrak{P}[q ; a]$.

Теорема 1. Для того чтобъ голоморфная в $\mathbb{D}$ функиия $v$ являлась инфинитезимальной образующей некоторой однопараметрической полугруппы в $\mathfrak{P}[q ; a], q \in \overline{\mathbb{D}}, a \in \mathbb{T}$, необходимо и достаточно, чтобы она допускала представление в виде

$$
v(z)=\alpha(q-z)(1-\bar{q} z)(1-\bar{a} z) h(\bar{a} z)
$$


где $\alpha>0$, а функиия $h$ определяется формулой

$$
h(z)=\int_{\mathbb{T}} \frac{1-\varkappa}{1-\varkappa z} d \mu(\varkappa)
$$

с некоторой вероятностной (регулярной, борелевской с единичной общей массой) мерой $\mu$ на $\mathbb{T}$.

Как отмечалось выше, с каждой однопараметрической полугруппой $t \mapsto f^{t}$ в $\mathfrak{P}[q], q \in \mathbb{D}$, однозначно связана функция Кёнигса $F$. Следующая теорема дает интегральное представление класса функций Кёнигса, отвечающих однопараметрическим полугруппам в $\mathfrak{P}$ с внутренней точкой Данжуа-Вольфа.

Теорема 2. Для того чтобы голоморфная в $\mathbb{D}$ функиия $F$ являлась функицей Кёнигса некоторой однопараметрической полугруппь в $\mathfrak{P}[q], q \in \mathbb{D}$, необходимо и достаточно, чтобы она допускала представление в виде

$$
F(z)=(z-q)\left(\frac{1-\bar{q} z}{1-|q|^{2}}\right)^{\sigma^{2}} \exp \left\{\left(1+\sigma^{2}\right) \int_{\mathbb{T}} \ln \frac{1-\varkappa q}{1-\varkappa z} d \mu(\varkappa)\right\}
$$

с некоторым $\sigma=e^{i \theta},-\pi / 2<\theta<\pi / 2$, и вероятностной мерой $\mu$ на $\mathbb{T}$. При этом под степенной функиией и логарифмом понимаются непрерывные ветви, которые принимают значения 1 и 0 соответственно при $z=q$.

Ответ на вопрос, как меняется вид функции Кёнигса, если кроме точки Данжуа-Вольфа $q \in \mathbb{D}$ для однопараметрической полугруппы $t \mapsto f^{t}$ неподвижной является также точка $a \in \mathbb{T}$, в которой функции $f^{t}, t>0$, имеют конечные угловые производные, дает следующий результат.

Теорема 3. Для того чтобы голоморфная в $\mathbb{D}$ функиия $F$ являлась функицей Кёнигса некоторой однопараметрической полугруппь в $\mathfrak{P}[q ; a], q \in \mathbb{D}$, $a \in \mathbb{T}$, необходимо и достаточно, чтобы она допускала представление в виде

$$
\begin{aligned}
F(z)=(z & -q)\left(\frac{1-\bar{q} z}{1-|q|^{2}}\right)^{\sigma^{2}}\left(\frac{1-\bar{a} q}{1-\bar{a} z}\right)^{\lambda_{1}\left(1+\sigma^{2}\right)} \\
& \times \exp \left\{\lambda_{2}\left(1+\sigma^{2}\right) \int_{\mathbb{T}} \ln \frac{1-\varkappa q}{1-\varkappa z} d \mu(\varkappa)\right\}
\end{aligned}
$$

с некоторыми $\sigma=e^{i \theta},-\pi / 2<\theta<\pi / 2, \lambda_{1}>0, \lambda_{2} \geqslant 0, \lambda_{1}+\lambda_{2}=1$, и вероятностной мерой $\mu$ на $\mathbb{T}$. При этом под степенными функииями и логарифмом понимаются непрерывные ветви, которые принимают значения 1 и 0 соответственно при $z=q$.

Пусть теперь $t \mapsto f^{t}$ - однопараметрическая полугруппа $t \mapsto f^{t}$ в $\mathfrak{P}$ с точкой Данжуа-Вольфа $q \in \mathbb{T}$. Тогда из формулы (1) видно, что инфинитезимальная образующая $v$ этой однопараметрической полугруппы не обращается в нуль в единичном круге. Поэтому аналитическая в $\mathbb{D}$ функция $F$ определяется однозначно с точностью до аддитивной константы посредством равенства $F^{\prime}(z)=1 / v(z)$. Нормируем ее условием $F(0)=0$ и будем называть функиией 
Кёнигса однопараметрической полугруппы $t \mapsto f^{t}$. Эта функция связана с однопараметрической полугруппой также равенством

$$
F\left(f^{t}(z)\right)=F(z)+t
$$

где $t \geqslant 0$, которое относительно $F$ является функциональным уравнением Абеля. Более подробно свойства функции Кёнигса $F$ обсуждаются в $§ 6$. Следующая теорема дает параметрическое представление класса функций Кёнигса, отвечающих однопараметрическим полугруппам с граничной точкой ДанжуаВольфа.

ТеОрема 4. Для того чтобы голоморфная в $\mathbb{D}$ функиия $F$ являлась функичей Кёнигса некоторой однопараметрической полугруппь в $\mathfrak{P}[q], q \in \mathbb{T}$, необходимо и достаточно, чтобы она допускала представление в виде

$$
\begin{aligned}
F(z)=i \beta & \frac{\bar{q} z}{1-\bar{q} z}+\lambda_{1} \frac{\bar{q} z}{(1-\bar{q} z)^{2}} \\
& +\lambda_{2} \int_{\mathbb{T} \backslash\{\bar{q}\}}\left(\ln \frac{1-\varkappa z}{1-\bar{q} z}+i(\operatorname{Im}\{\varkappa q\}) \frac{\bar{q} z}{1-\bar{q} z}\right) \frac{d \mu(\varkappa)}{1-\operatorname{Re}\{\varkappa q\}}
\end{aligned}
$$

с некоторыми $\beta \in \mathbb{R}, \lambda_{1}, \lambda_{2} \geqslant 0$ и вероятностной мерой $\mu$ на $\mathbb{T} \backslash\{\bar{q}\}$. При этом под логарифмом понимается непрерывная ветвъ, обращающаяся в нуль при $z=0$.

Наличие дополнительной неподвижной точки, в которой функции однопараметрической полугруппы имеют конечные угловые производные, уточняет формулу (6) следующим образом.

Теорема 5. Для того чтобы голоморфная в $\mathbb{D}$ функиия $F$ являласъ функцией Кёнигса некоторой однопараметрической полугруппъ в $\mathfrak{P}[q ; a], q \in \mathbb{T}$, $a \in \mathbb{T}$, необходимо и достаточно, чтобы она допускала представление в виде

$$
\begin{aligned}
F(z)=i \beta & \frac{\bar{q} z}{1-\bar{q} z}+\lambda_{1} \ln \frac{1-\bar{a} z}{1-\bar{q} z}+\lambda_{2} \frac{\bar{q} z}{(1-\bar{q} z)^{2}} \\
& +\lambda_{3} \int_{\mathbb{T} \backslash\{\bar{q}\}}\left(\ln \frac{1-\varkappa z}{1-\bar{q} z}+i(\operatorname{Im}\{\varkappa q\}) \frac{\bar{q} z}{1-\bar{q} z}\right) \frac{d \mu(\varkappa)}{1-\operatorname{Re}\{\varkappa q\}}
\end{aligned}
$$

с некоторыми $\beta \in \mathbb{R}, \lambda_{1}>0, \lambda_{2}, \lambda_{3} \geqslant 0$ и вероятностной мерой $\mu$ на $\mathbb{T} \backslash\{\bar{q}\}$. При этом под логарифмами понимаются непрерывные ветви, обращающиеся в нуль при $z=0$.

\section{§ 3. Некоторые предварительные сведения и результаты}

Основные сведения и результаты, связанные с гиперболической геометрией в единичном круге и угловой производной, которые понадобятся нам в дальнейшем, можно найти, например, в монографиях [10] и [11].

В связи с описанием инфинитезимальных образующих однопараметрических полугрупп в $\mathfrak{P}[q ; a]$ естественно возникает класс $\mathscr{Q}$ функций $h$, голоморфных в единичном круге и допускающих представление в виде (3) с некоторой вероятностной мерой $\mu$ на $\mathbb{T}$. Приведем два эквивалентных определения класса $\mathscr{Q}$ и изучим некоторые свойства функций этого класса. 
Лемма 1. Для того чтобы голоморфная в $\mathbb{D}$ функиия $h$ принадлежала классу $\mathscr{Q}$, необходимо и достаточно, чтобъ она допускала представление в виде

$$
h(z)=\frac{1-\varphi(z)}{1-z \varphi(z)},
$$

где $\varphi$ - голоморфная в $\mathbb{D}$ функиия, удовлетворяющая условию $|\varphi(z)| \leqslant 1$ при $z \in \mathbb{D}$.

ДокАЗАТЕЛьство. Пусть $h \in \mathscr{Q}$ и имеет место представление (3) с вероятностной мерой на $\mathbb{T}$. Замечая, что

$$
\frac{1-\varkappa}{1-\varkappa z}=\frac{1}{2}\left[\left(\frac{1+\varkappa z}{1-\varkappa z}+1\right)-\frac{1}{z}\left(\frac{1+\varkappa z}{1-\varkappa z}-1\right)\right],
$$

приходим к равенству

$$
h(z)=\frac{1}{2}\left[(p(z)+1)-\frac{1}{z}(p(z)-1)\right],
$$

где

$$
p(z)=\int_{\mathbb{T}} \frac{1+\varkappa z}{1-\varkappa z} d \mu(\varkappa) .
$$

Поскольку $p$ голоморфна в $\mathbb{D}$, имеет положительную вещественную часть и $p(0)=1$, то функция

$$
\psi(z)=\frac{p(z)-1}{p(z)+1}
$$

удовлетворяет условиям леммы Шварца, т.е. $|\psi(z)| \leqslant 1$ при $z \in \mathbb{D}$ и $\psi(0)=0$. Но тогда $\varphi(z)=\psi(z) / z$ также является голоморфной в $\mathbb{D}$ функцией и удовлетворяет условию $|\varphi(z)| \leqslant 1$ при $z \in \mathbb{D}$. Выражая теперь функцию $p$ через $\varphi$ и подставляя полученное выражение в (9), приходим к равенству (8).

Обратно, пусть $h$ имеет представление $(8)$ с некоторой голоморфной в $\mathbb{D}$ функцией $\varphi$, удовлетворяющей условию $|\varphi(z)| \leqslant 1$ при $z \in \mathbb{D}$. Полагая

$$
p(z)=\frac{1+z \varphi(z)}{1-z \varphi(z)}
$$

получаем голоморфную в $\mathbb{D}$ функцию с положительной вещественной частью и удовлетворяющую условию $p(0)=1$. По теореме Рисса-Герглотца найдется такая вероятностная мера $\mu$ на $\mathbb{T}$, которая связана с функцией $p$ равенством (10). Но тогда

$$
\begin{aligned}
h(z) & =\frac{1-\varphi(z)}{1-z \varphi(z)}=\frac{1}{2}\left[\left(\frac{1+z \varphi(z)}{1-z \varphi(z)}+1\right)-\frac{1}{z}\left(\frac{1+z \varphi(z)}{1-z \varphi(z)}-1\right)\right] \\
& =\frac{1}{2}\left[(p(z)+1)-\frac{1}{z}(p(z)-1)\right]=\int_{\mathbb{T}} \frac{1-\varkappa}{1-\varkappa z} d \mu(\varkappa)
\end{aligned}
$$

и лемма доказана.

ЗАмечАниЕ 1. Из представления (8) следует, что либо функция $h$ класса $\mathscr{Q}$ тождественно обращается в нуль, либо $h(z) \neq 0$ при $z \in \mathbb{D}$. 
Лемма 2. Пусть $h$ - голоморфная в $\mathbb{D}$ функиия и $h(z) \not \equiv 0$. Тогда $h$ принадлежит классу $\mathscr{Q}$ в том и только том случае, если имеет место представление

$$
\frac{1}{h(z)}=\frac{1}{2}(1-z)\left[\frac{1+z}{1-z}+g(z)\right]
$$

где $g$ - голоморфная с неотрицательной вещественной частъю в $\mathbb{D}$ функиия.

Доказательство. Пусть $h \in \mathscr{Q}$ и $h(z) \not \equiv 0$. В силу леммы 1 функция $h$ допускает представление $(8)$ с некоторой голоморфной в $\mathbb{D}$ функцией $\varphi$, удовлетворяющей условиям $|\varphi(z)| \leqslant 1$ при $z \in \mathbb{D}$ и $\varphi(z) \not \equiv 1$. Поэтому

$$
\begin{aligned}
\frac{1}{h(z)} & =\frac{1-z \varphi(z)}{1-\varphi(z)}=\frac{1}{1-\varphi(z)}-z \frac{\varphi(z)}{1-\varphi(z)} \\
& =\frac{1}{2}\left[\left(\frac{1+\varphi(z)}{1-\varphi(z)}+1\right)-z\left(\frac{1+\varphi(z)}{1-\varphi(z)}-1\right)\right]=\frac{1}{2}(1-z)\left[\frac{1+z}{1-z}+g(z)\right],
\end{aligned}
$$

где $g(z)=(1+\varphi(z)) /(1-\varphi(z))$.

Обратно, пусть имеет место представление (11), тогда функция $\varphi(z)=$ $(g(z)-1) /(g(z)+1)$ будет голоморфной в $\mathbb{D}$ и $|\varphi(z)| \leqslant 1$ при $z \in \mathbb{D}$. При этом $g(z)=(1+\varphi(z)) /(1-\varphi(z))$ и

$$
h(z)=\frac{2}{(1-z)\left(\frac{1+z}{1-z}+\frac{1+\varphi(z)}{1-\varphi(z)}\right)}=\frac{1-\varphi(z)}{1-z \varphi(z)},
$$

т.е. $h$ принадлежит классу $\mathscr{Q}$ в силу леммы 1.

СледСтвиЕ 1. Для любой функиии $h$ класса $\mathscr{Q}$ выполняется неравенство

$$
\operatorname{Re}\{(1-z) h(z)\} \geqslant 0
$$

при всех $z \in \mathbb{D}$.

Лемма 3. Пусть $z_{0}$ - фиксированная точка единичного круга $\mathbb{D}$. Тогда множество

$$
K_{z_{0}}=\left\{w=h\left(z_{0}\right): h \in \mathscr{Q}\right\}
$$

представляет собой замкнутый круг

$$
\left\{w:\left|w-\frac{1-\bar{z}_{0}}{1-\left|z_{0}\right|^{2}}\right| \leqslant \frac{\left|1-z_{0}\right|}{1-\left|z_{0}\right|^{2}}\right\} .
$$

При этом граничную точку $\zeta \in \partial K_{z_{0}}$ вносит единственная функчия класса $\mathscr{Q}$, определяемая равенствами

$$
h(z)=\frac{1-\varkappa}{1-\varkappa z}, \quad \varkappa=\frac{1-\zeta}{1-\zeta z_{0}} .
$$

ДокАЗАтЕльство. Непосредственно из формулы (3), определяющей класс $\mathscr{Q}$, следует, что множество $K_{z_{0}}$ представляет собой замкнутую выпуклую оболочку кривой $\Gamma_{z_{0}}$, которая является образом единичной окружности $\mathbb{T}$ при дробно-линейном преобразовании

$$
L(\zeta)=\frac{1-\zeta}{1-z_{0} \zeta}
$$


Из кругового свойства дробно-линейных отображений следует, что $\Gamma_{z_{0}}-$ окружность. Ее центр $w_{0}$ в силу принципа симметрии является образом точки $\bar{z}_{0}$, которая симметрична относительно единичной окружности $\mathbb{T}$ полюсу $1 / z_{0}$ дробно-линейного преобразования $L$. Следовательно,

$$
w_{0}=L\left(\bar{z}_{0}\right)=\frac{1-\bar{z}_{0}}{1-\left|z_{0}\right|^{2}} .
$$

Замечая также, что $\Gamma_{z_{0}}$ проходит через начало координат, делаем вывод, что радиус этой окружности равняется $\left|w_{0}\right|$.

Граничную точку $\zeta$ круга $K_{z_{0}}$ вносит функция $h$, которой в представлении (3) соответствует атомарная мера $\mu$, сосредоточенная в точке $\varkappa \in \mathbb{T}$, определяемой из равенства $\zeta=L(\varkappa)$. Лемма доказана.

СлеДСтвиЕ 2. Для любой функции $h$ из класса $\mathscr{Q}$ выполняется неравенство

$$
|h(z)| \leqslant \frac{2|1-z|}{1-|z|^{2}}
$$

при всех $z \in \mathbb{D}$.

\section{§ 4. Аналог формулы Берксона-Порты}

ДокаЗАтеЛьСтво теоремы 1. Необходимость. Пусть $t \mapsto f^{t}-$ нетривиальная $\left(f^{t}(z) \not \equiv z\right.$ при $\left.t>0\right)$ однопараметрическая полугруппа в $\mathfrak{P}[q ; a]$ и

$$
v(z)=\left.\frac{\partial}{\partial t} f^{t}(z)\right|_{t=0}
$$

- ее инфинитезимальная образующая. Нам нужно доказать, что $v$ можно представить в виде $(2)$ с некоторой функцией $h$ из класса $\mathscr{Q}$.

Допустим вначале, что $q=0$ и $a=1$. Для любой функции $f \in \mathfrak{P}[0 ; 1]$ выполняется неравенство $f^{\prime}(1) \geqslant 1$ и знак равенства достигается лишь в случае $f(z) \equiv z$ (см., например, [5] или [10]). Поэтому из условия $f^{t+s}(z)=f^{t} \circ f^{s}(z)$ и свойств угловой производной следует, что функция

$$
\beta(t)=\left(f^{t}\right)^{\prime}(1)
$$

является возрастающей и удовлетворяет условиям

$$
\beta(t+s)=\beta(t) \beta(s), \quad \beta(0)=1 .
$$

Следовательно, $\beta(t)=e^{\lambda t}$, где $\lambda=\ln \left(f^{1}\right)^{\prime}(1)>0$.

Рассмотрим семейство дробно-линейных преобразований

$$
U_{t}(z)=\frac{z+u(t)}{1+u(t) z}, \quad u(t)=\frac{e^{\lambda t}-1}{e^{\lambda t}+1},
$$

где $t>0$, которые отображают единичный круг $\mathbb{D}$ на себя и оставляют неподвижными точки $z= \pm 1$. Полагая

$$
g_{t}(z)=f^{t} \circ U_{t}(z)
$$


получаем семейство $\left\{g_{t}\right\}_{t \geqslant 0}$ отображений единичного круга $\mathbb{D}$ в себя, которое дифференцируемо по $t$, и $g_{0}(z) \equiv z$. Кроме того, при каждом $t>0$ функция $g_{t}$ оставляет неподвижной точку $z=1$ и в этой точке имеет угловую производную $g_{t}^{\prime}(1)=1$. В силу теоремы Жюлиа-Каратеодори образ $g_{t}(z)$ точки $z \in \mathbb{D}$ попадает внутрь орицикла, на границе которого лежит $z$, т.е. внутрь круга с центром в точке

$$
\xi=\frac{1-|z|^{2}}{2(1-\operatorname{Re} z)}
$$

и радиуса

$$
r=\frac{|1-z|^{2}}{2(1-\operatorname{Re} z)} .
$$

Отмеченное обстоятельство означает, что угол между векторами $\xi-z$ и $g_{t}(z)-z$ не должен превышать $\pi / 2$. Замечая также, что

$$
\begin{aligned}
\arg \left\{\frac{1-|z|^{2}}{2(1-\operatorname{Re} z)}-z\right\} & =\arg \left\{1-|z|^{2}-2 z(1-\operatorname{Re} z)\right\} \\
& =\arg \{1-z \bar{z}-z((1-z)+(1-\bar{z}))\}=\arg \left\{(1-z)^{2}\right\},
\end{aligned}
$$

приходим к условию

$$
\operatorname{Re} \frac{g_{t}(z)-z}{(1-z)^{2}}>0
$$

при $z \in \mathbb{D}$. Отсюда видно, что

$$
\left.\frac{\partial}{\partial t} g_{t}(z)\right|_{t=0}=\lim _{t \rightarrow 0} \frac{g_{t}(z)-z}{t}=(1-z)^{2} p(z),
$$

где $p$ - голоморфная в $\mathbb{D}$ функция с неотрицательной вещественной частью.

С другой стороны,

$$
\begin{aligned}
\left.\frac{\partial}{\partial t} g_{t}(z)\right|_{t=0} & =\left.\frac{\partial}{\partial t} f^{t}\left(U_{t}(z)\right)\right|_{t=0}+\left.\left(f^{t}\right)^{\prime}\left(U_{t}(z)\right) \frac{\partial}{\partial t} U_{t}(z)\right|_{t=0} \\
& =v(z)+\frac{\lambda}{2}\left(1-z^{2}\right)
\end{aligned}
$$

где $v$ - инфинитезимальная образующая однопараметрической полугруппы $t \mapsto f^{t}$.

Таким образом, мы приходим к равенству

$$
v(z)=(1-z)\left[(1-z) p(z)-\frac{\lambda}{2}(1+z)\right] .
$$

Поскольку мы предполагали, что $q=0$, т.е. точка $z=0$ является неподвижной для функций $f^{t}, t>0$, то $v(0)=0$. Но тогда $p(0)=\lambda / 2$ и функция

$$
p_{1}(z)=\frac{2}{\lambda} p(z)
$$

имеет в $\mathbb{D}$ положительную вещественную часть и нормирована условием $p_{1}(0)=1$. По теореме Рисса-Герглотца найдется такая вероятностная мера $\mu$ на $\mathbb{T}$, что

$$
p_{1}(z)=\int_{\mathbb{T}} \frac{1+\varkappa z}{1-\varkappa z} d \mu(\varkappa) .
$$


Это позволяет представить инфинитезимальную образующую $v$ в виде

$$
\begin{aligned}
v(z) & =\frac{\lambda}{2}(1-z)\left[(1-z) \int_{\mathbb{T}} \frac{1+\varkappa z}{1-\varkappa z} d \mu(\varkappa)-(1+z)\right] \\
& =-\lambda z(1-z) \int_{\mathbb{T}} \frac{1-\varkappa}{1-\varkappa z} d \mu(\varkappa) .
\end{aligned}
$$

Полученное представление инфинитезимальной образующей $v$ совпадает с приведенным в формулировке теоремы в случае, когда $q=0$ и $a=1$.

Допустим теперь, что $t \mapsto f^{t}$ - однопараметрическая полугруппа в $\mathfrak{P}[q ; a]$, $q \in \mathbb{D}, a \in \mathbb{T}$, т.е. точка Данжуа-Вольфа $q$ является внутренней и все функции $f^{t}, t>0$, оставляют также неподвижной точку $a \in \mathbb{T}$, в которой имеют конечные угловые производные. Введем в рассмотрение дробно-линейное преобразование

$$
L(z)=\frac{1-\bar{q} a}{a-q} \frac{z-q}{1-\bar{q} z},
$$

которое отображает единичный круг $\mathbb{D}$ на себя и удовлетворяет условиям $L(q)=0, L(a)=1$. Для каждого $t \geqslant 0$ определим

$$
\tilde{f}^{t}(\zeta)=L \circ f^{t} \circ L^{-1}(\zeta)
$$

Очевидно, что $t \mapsto \widetilde{f^{t}}$ является однопараметрической полугруппой в $\mathfrak{P}[0 ; 1]$. Поэтому по доказанному ее инфинитезимальная образующая

$$
\widetilde{v}(\zeta)=\left.\frac{\partial}{\partial t} \widetilde{f}^{t}(\zeta)\right|_{t=0}
$$

допускает представление в виде

$$
\widetilde{v}(\zeta)=-\alpha \zeta(1-\zeta) \int_{\mathbb{T}} \frac{1-\varkappa}{1-\varkappa \zeta} d \mu(\varkappa),
$$

где $\alpha>0$, а $\mu$ - вероятностная мера на $\mathbb{T}$. С другой стороны,

$$
\widetilde{v}(L(z))=\left.\frac{\partial}{\partial t} L\left(f^{t}(z)\right)\right|_{t=0}=L^{\prime}(z) v(z)
$$

где $v$ - инфинитезимальная образующая однопараметрической полугруппы $t \mapsto f^{t}$. Следовательно,

$$
v(z)=-\alpha \frac{L(z)(1-L(z))}{L^{\prime}(z)} \int_{\mathbb{T}} \frac{1-\varkappa}{1-\varkappa L(z)} d \mu(\varkappa) .
$$

Замечая, что

$$
\frac{L(z)(1-L(z))}{L^{\prime}(z)}=\frac{(z-q)(1-\bar{a} z)}{1-\bar{a} q}, \quad \frac{1-\varkappa}{1-\varkappa L(z)}=\frac{1-\bar{q} z}{1-\bar{a} q} \frac{1-\eta}{1-\eta \bar{a} z},
$$

где

$$
\eta=\frac{(1-a \bar{q}) \varkappa+\bar{q}(a-q)}{(1-\bar{a} q)+q(\bar{a}-\bar{q}) \varkappa}
$$


пробегает единичную окружность $\mathbb{T}$ вместе с $\varkappa$, приходим к равенству

$$
v(z)=\frac{\alpha}{|1-\bar{a} q|^{2}}(q-z)(1-\bar{q} z)(1-\bar{a} z) \int_{\mathbb{T}} \frac{1-\eta}{1-\eta \bar{a} z} d \nu(\eta),
$$

в котором $\nu$ - вероятностная мера на $\mathbb{T}$. Таким образом, в случае внутренней точки Данжуа-Вольфа однопараметрическая полугруппа $t \mapsto f^{t}$ в $\mathfrak{P}[q ; a]$ имеет инфинитезимальную образующую $v$, вид которой определяется формулами $(2),(3)$.

Приступая к рассмотрению случая граничной точки Данжуа-Вольфа, допустим вначале, что $t \mapsto f^{t}-$ нетривиальная однопараметрическая полугруппа в $\mathfrak{P}[-1 ; 1]$, т.е. функции $f^{t}, t>0$, имеют точку Данжуа-Вольфа $q=-1$ и оставляют неподвижной точку $a=1$, в которой конечны угловые производные $\left(f^{t}\right)^{\prime}(1)$. В силу формулы Берксона-Порты (1) инфинитезимальная образующая $v$ этой однопараметрической полугруппы допускает представление в виде

$$
v(z)=-(1+z)^{2} p_{1}(z),
$$

где $p_{1}$ - голоморфная в $\mathbb{D}$ функция с положительной вещественной частью. Однако эта формула не учитывает того, что функции $f^{t}$ имеют кроме $q=-1$ также неподвижную точку $a=1$.

Снова, как и в случае $q=0, a=1$, выполняется равенство $\left(f^{t}\right)^{\prime}(1)=e^{\lambda t}$, $t \geqslant 0$, с некоторым $\lambda>0$. Поэтому, определяя семейство функций $g_{t}(z)=$ $f^{t} \circ U_{t}(z), t \geqslant 0$, получаем

$$
\left.\frac{\partial}{\partial t} g_{t}(z)\right|_{t=0}=(1-z)^{2} p_{2}(z)
$$

где $p_{2}$ также является голоморфной в $\mathbb{D}$ функцией с положительной вещественной частью. Вычисление этой производной другим способом приводит к равенству

$$
(1-z)^{2} p_{2}(z)=v(z)+\frac{\lambda}{2}\left(1-z^{2}\right),
$$

которое с учетом вида инфинитезимальной образующей $v$ можно переписать следующим образом:

$$
(1-z)^{2} p_{2}(z)=\frac{\lambda}{2}\left(1-z^{2}\right)-(1+z)^{2} p_{1}(z) .
$$

Полагая в последнем равенстве $z=0$, получаем

$$
p_{1}(0)+p_{2}(0)=\frac{\lambda}{2}
$$

Это означает, что к функции

$$
p(z)=\frac{2}{\lambda}\left(p_{1}(z)+p_{2}(z)\right)
$$

применима теорема Рисса-Герглотца, согласно которой

$$
p(z)=\int_{\mathbb{T}} \frac{1+\varkappa z}{1-\varkappa z} d \mu(\varkappa),
$$


где $\mu$ - вероятностная мера на $\mathbb{T}$. Далее, используя полученные выше равенства, выполним следующие преобразования:

$$
\begin{aligned}
v(z) & =(1-z)\left[(1-z) p_{2}(z)-\frac{\lambda}{2}(1+z)\right] \\
& =(1-z)\left[(1-z)\left(p_{2}(z)+p_{1}(z)\right)-\frac{\lambda}{2}(1+z)\right]-(1-z)^{2} p_{1}(z) \\
& =\frac{\lambda}{2}(1-z)[(1-z) p(z)-(1+z)]+\left(\frac{1-z}{1+z}\right)^{2} v(z) .
\end{aligned}
$$

Следовательно,

$$
\left[1-\left(\frac{1-z}{1+z}\right)^{2}\right] v(z)=-\lambda z(1-z) \int_{\mathbb{T}} \frac{1-\varkappa}{1-\varkappa z} d \mu(\varkappa),
$$

и после очевидных преобразований получаем

$$
v(z)=-\frac{\lambda}{4}(1+z)^{2}(1-z) \int_{\mathbb{T}} \frac{1-\varkappa}{1-\varkappa z} d \mu(\varkappa) .
$$

Таким образом, в случае $q=-1$ и $a=1$ мы снова получили вид инфинитезимальной образующей, описанный формулами (2), (3).

Остается рассмотреть случай, когда однопараметрическая полугруппа $t \mapsto f^{t}$ имеет точку Данжуа-Вольфа $q$ и неподвижную точку $a$, произвольно расположенные на единичной окружности $\mathbb{T}$. Используя дробно-линейное преобразование

$$
L(z)=\frac{q-(2-a \bar{q}) z}{(q-2 a)+a \bar{q} z},
$$

которое отображает единичный круг $\mathbb{D}$ на себя и удовлетворяет условиям $L(q)=-1, L(a)=1$, определим семейство функций:

$$
\widetilde{f}^{t}(\zeta)=L \circ f^{t} \circ L^{-1}(\zeta)
$$

где $t \geqslant 0$. Легко видеть, что $t \mapsto \widetilde{f}^{t}$ является однопараметрической полугруппой в $\mathfrak{P}[-1 ; 1]$. По доказанному инфинитезимальная образующая однопараметрической полугруппы $t \mapsto \widetilde{f}^{t}$ имеет вид

$$
\widetilde{v}(\zeta)=\left.\frac{\partial}{\partial t} \tilde{f}^{t}(\zeta)\right|_{t=0}=-\alpha(1+\zeta)^{2}(1-\zeta) \int_{\mathbb{T}} \frac{1-\varkappa}{1-\varkappa \zeta} d \mu(\varkappa),
$$

где $\alpha>0$, а $\mu$ - вероятностная мера на $\mathbb{T}$. Но тогда инфинитезимальная образующая $v$ однопараметрической полугруппы $t \mapsto f^{t}$ будет определяться равенством

$$
v(z)=-\alpha \frac{(1+L(z))^{2}(1-L(z))}{L^{\prime}(z)} \int_{\mathbb{T}} \frac{1-\varkappa}{1-\varkappa L(z)} d \mu(\varkappa) .
$$

Замечая, что

$$
\begin{gathered}
\frac{(1+L(z))^{2}(1-L(z))}{L^{\prime}(z)}=\frac{4 \alpha(q-z)(1-\bar{q} z)(1-\bar{a} z)}{(q-2 a)+a \bar{q} z}, \\
\frac{1-\varkappa}{1-\varkappa L(z)}=-\frac{(q-2 a)+a \bar{q} z}{a|q-a|^{2}} \frac{1-\eta}{1-\eta \bar{a} z},
\end{gathered}
$$


где

$$
\eta=\frac{a \bar{q}+(2-a \bar{q}) \varkappa}{\bar{a} q \varkappa+(2-\bar{a} q)}
$$

пробегает единичную окружность $\mathbb{T}$ вместе с $\varkappa$, приходим к равенству

$$
v(z)=\frac{4 \alpha}{|q-a|^{2}}(q-z)(1-\bar{q} z)(1-\bar{a} z) \int_{\mathbb{T}} \frac{1-\eta}{1-\eta \bar{a} z} d \nu(\eta),
$$

где $\nu$ - вероятностная мера на $\mathbb{T}$.

В результате мы установили, что при любых $q \in \overline{\mathbb{D}}$ и $a \in \mathbb{T}$ вид инфинитезимальной образующей $v$ однопараметрической полугруппы $t \mapsto f^{t}$ в $\mathfrak{P}[q ; a]$ определяется формулами (2), (3).

Достаточность. Допустим теперь, что функция $v$ имеет вид, определяемый формулами (2), (3), т.е.

$$
v(z)=\alpha(q-z)(1-\bar{q} z)(1-\bar{a} z) h(\bar{a} z),
$$

где $\alpha>0, q \in \overline{\mathbb{D}}, a \in \mathbb{T}$ и $h-$ функция класса $\mathscr{Q}$. В силу следствия 1 выполняется неравенство

$$
\operatorname{Re}\{(1-\bar{a} z) h(\bar{a} z)\} \geqslant 0
$$

при $z \in \mathbb{D}$. Это означает, что $v$ можно рассматривать как инфинитезимальную образующую некоторой однопараметрической полугруппы $t \mapsto f^{t}$ в $\mathfrak{P}[q]$. Остается показать, что функции $f^{t}, t>0$, оставляют неподвижной точку $a$ и имеют в ней конечные угловые производные.

Покажем вначале, что сама инфинитезимальная образующая $v$ имеет конечную угловую производную $v^{\prime}(a)$ в точке $a$. Для $M>1$ через

$$
\Delta_{M}(a)=\left\{z \in \mathbb{D}: \frac{\left|a^{2}-z^{2}\right|}{1-|z|^{2}}<M\right\}
$$

обозначим круговую луночку с вершинами в точках $a$ и $-a$. С точки зрения гиперболической геометрии в круге $\mathbb{D}$, которая обычно определяется элементом длины

$$
d s=\frac{2|d z|}{1-|z|^{2}},
$$

граница $\partial \Delta_{M}(a)$ этой луночки представляет собой множество точек, равноудаленных от диаметра круга $\mathbb{D}$ с концевыми точками $a$ и $-a$. Через

$$
\Delta_{M}^{+}(a)=\left\{z \in \Delta_{M}(a): \operatorname{Re}\{\bar{a} z\}>0\right\}
$$

будем обозначать половину луночки $\Delta_{M}(a)$, которая примыкает к точке $a$. Используя неравенство (12) для функций класса $\mathscr{Q}$, получаем

$$
\left|\frac{1-\varkappa}{1-\varkappa \bar{a} z}\right| \leqslant \frac{2|1-\bar{a} z|}{1-|z|^{2}}=\frac{2}{|1+\bar{a} z|} \frac{\left|a^{2}-z^{2}\right|}{1-|z|^{2}} \leqslant 2 M
$$

при всех $\varkappa \in \mathbb{T}$ и $z \in \Delta_{M}^{+}(a)$. Поэтому в интеграле, определяющем функцию $h$, можно осуществить предельный переход при $z \rightarrow a, z \in \Delta_{M}^{+}(a)$, в результате чего получим

$$
\lim _{z \rightarrow a} h(\bar{a} z)=\lim _{z \rightarrow a} \int_{\mathbb{T}} \frac{1-\varkappa}{1-\varkappa \bar{a} z} d \mu(\varkappa)=\mu(\mathbb{T} \backslash\{1\}) .
$$


Отсюда следует, что при $z \rightarrow a, z \in \Delta_{M}^{+}(a)$, имеют место предельные соотношения

$$
\lim _{z \rightarrow a} v(z)=0, \quad \lim _{z \rightarrow a} \frac{v(z)}{z-a}=\alpha|1-\bar{q} a|^{2} \mu(\mathbb{T} \backslash\{1\})=v^{\prime}(a) .
$$

Таким образом, угловая производная $v^{\prime}(a)$ существует и конечна. Кроме того, $v^{\prime}(a)>0$, если только мера $\mu$ не сосредоточена в одной точке $\varkappa=1$. Последний случай соответствует тому, что $v(z) \equiv 0$ и $f^{t}(z) \equiv z$ при всех $t \geqslant 0$. Поэтому в дальнейшем будем предполагать, что $v^{\prime}(a)>0$.

Пусть $R_{a}=\{z=r a: 1 / 2<r<1\}$ - половина радиуса с концевой точкой $a$. Фиксируем $M>1$ и выберем $\delta>0$ так, чтобы оно было меньше гиперболического расстояния от $R_{a}$ до границы $\partial \Delta_{M}^{+}(a)$ области $\Delta_{M}^{+}(a)$. Для каждого $z \in R_{a}$ через

$$
\tau_{z}=\inf \left\{t>0: f^{t}(z) \in \partial \Delta_{M}^{+}(a)\right\}
$$

обозначим момент первого выхода точки $f^{t}(z)$ на границу области $\Delta_{M}^{+}(a)$. Через $\gamma_{z}$ обозначим кривую с параметризацией $\zeta(t)=f^{t}(z), 0 \leqslant t<\tau_{z}$. Используя неравенство (12), для $z \in \Delta_{M}^{+}(a)$ получаем

$$
\frac{|v(z)|}{1-|z|^{2}} \leqslant \frac{4 \alpha|a-z||h(\bar{a} z)|}{1-|z|^{2}} \leqslant \frac{8 \alpha|a-z||1-\bar{a} z|}{\left(1-|z|^{2}\right)^{2}} \leqslant 8 \alpha \frac{\left|a^{2}-z^{2}\right|^{2}}{\left(1-|z|^{2}\right)^{2}} \leqslant 8 \alpha M^{2} .
$$

Но тогда гиперболическая длина $l_{H}\left(\gamma_{z}\right)$ кривой $\gamma_{z}$ будет удовлетворять неравенству

$$
l_{H}\left(\gamma_{z}\right)=2 \int_{\gamma_{z}} \frac{|d \zeta|}{1-|\zeta|^{2}}=2 \int_{0}^{\tau_{z}} \frac{\left|v\left(f^{t}(z)\right)\right|}{1-\left|f^{t}(z)\right|^{2}} d t \leqslant 16 \alpha M^{2} \tau_{z}
$$

В силу условий выбора $\delta$ для всех $z \in R_{a}$ получаем

$$
\delta \leqslant l_{H}\left(\gamma_{z}\right) \leqslant 16 \alpha M^{2} \tau_{z}
$$

откуда следует неравенство

$$
\tau_{z} \geqslant \frac{\delta}{16 \alpha M^{2}}=\tau^{*}
$$

Таким образом, если $t \in\left[0, \tau^{*}\right]$ и $z \in R_{a}$, то гиперболическое расстояние от $z$ до $f^{t}(z)$ не превышает $16 \alpha M^{2} \tau^{*}=\delta$. Следовательно, при всех $t \in\left[0, \tau^{*}\right]$ образ $f^{t}\left(R_{a}\right)$ радиального отрезка $R_{a}$ содержится в $\Delta_{M}^{+}(a)$ и $f^{t}(z) \rightarrow a$ при $z \rightarrow a$ вдоль $R_{a}$.

Далее, поскольку

$$
\frac{\partial}{\partial t} \ln \frac{f^{t}(z)-a}{z-a}=\frac{v\left(f^{t}(z)\right)}{f^{t}(z)-a}
$$

где под логарифмом понимается непрерывная ветвь, обращающаяся в нуль при $t=0$, то

$$
\ln \frac{f^{t}(z)-a}{z-a}=\int_{0}^{t} \frac{v\left(f^{s}(z)\right)}{f^{s}(z)-a} d s
$$


Как было показано выше, инфинитезимальная образующая $v$ имеет в точке $a$ конечный угловой предел $v^{\prime}(a)>0$. Поэтому, осуществляя в последнем равенстве предельный переход при $z \rightarrow a$ вдоль отрезка $R_{a}$, получаем

$$
\lim _{z \rightarrow a} \ln \frac{f^{t}(z)-a}{z-a}=v^{\prime}(a) t .
$$

Это означает, что функции $f^{t}, 0<t \leqslant \tau^{*}$, имеют в точке $a$ угловую производную и

$$
\left(f^{t}\right)^{\prime}(a)=e^{t v^{\prime}(a)}
$$

В силу полугруппового свойства $f^{t+s}(z)=f^{t} \circ f^{s}(z)$ существование угловой производной в точке $a$ и полученное для нее равенство распространяются на все функции $f^{t}, t>0$, однопараметрической полугруппы. Этим завершается доказательство теоремы 1.

\section{§5. Функция Кёнигса в случае внутренней точки Данжуа-Вольфа}

Как уже отмечалось в $\S 1$, история проблемы дробных итераций восходит к работам Шрёдера [1] и Кёнигса [2]. Следует также отметить, что они рассматривали эту проблему в локальной постановке, т.е. когда функция и ее итерации определены в окрестности неподвижной точки. В частности, Шрёдер связал проблему дробных итераций с решением функциональных уравнений. Позже это направление получило развитие в многочисленных работах различных авторов (см., например, [12] и приведенную там библиографию). Кёнигс предложил конструкцию из натуральных итераций и показал, что она сходится к непостоянной аналитической функции, которая является решением уравнения Шрёдера. Вопросы сходимости нормированных последовательностей итераций аналитических функций в круге (или полуплоскости) подробно рассмотрены в монографии [6]. В частности, если $f \in \mathfrak{P}$ отлична от дробно-линейного преобразования единичного круга $\mathbb{D}$ на себя и имеет внутреннюю точку Данжуа-Вольфа $q \in \mathbb{D}$, в которой $f^{\prime}(q) \neq 0$, то существует локально равномерный в $\mathbb{D}$ предел

$$
\lim _{n \rightarrow \infty} \frac{f^{n}(z)-q}{\left(f^{\prime}(q)\right)^{n}}=F(z) .
$$

Очевидно, что $F$ является аналитической в $\mathbb{D}$ функцией и $F(q)=0, F^{\prime}(q)=1$. Ее называют функцией Кёнигса и она является решением уравнения Шрёдера

$$
F \circ f(z)=f^{\prime}(q) F(z)
$$

Легко видеть, что и все натуральные итерации $f^{n}, n=2,3, \ldots$, функции $f$ имеют ту же самую функцию Кёнигса $F$.

Допустим теперь, что $t \mapsto f^{t}$ - однопараметрическая полугруппа в $\mathfrak{P}[q]$, $q \in \mathbb{D}$. Тогда функции $f^{t}, t>0$, будут однолистными в $\mathbb{D}$, и для них будет определена функция Кёнигса

$$
F(z)=\lim _{t \rightarrow \infty} \frac{f^{t}(z)-q}{\left(f^{t}\right)^{\prime}(q)} .
$$


Заметим, что в этом случае функция $F$ как предел последовательности однолистных функций также будет однолистной. При этом для всех $t \geqslant 0$ будет выполняться соотношение

$$
F \circ f^{t}(z)=\left(f^{t}\right)^{\prime}(q) F(z)
$$

Если $v$ - инфинитезимальная образующая однопараметрической полугруппы $t \mapsto f^{t}$, то, дифференцируя последнее равенство по $t$, получаем

$$
F^{\prime}\left(f^{t}(z)\right) v\left(f^{t}(z)\right)=v^{\prime}(q) e^{t v^{\prime}(q)} F(z)
$$

Здесь мы воспользовались равенством $\left(f^{t}\right)^{\prime}(q)=e^{v^{\prime}(q) t}$, которое получается в результате интегрирования дифференциального уравнения

$$
\frac{d}{d t}\left(f^{t}\right)^{\prime}(q)=v^{\prime}(q)\left(f^{t}\right)^{\prime}(q)
$$

с начальным условием $\left.\left(f^{t}\right)^{\prime}(q)\right|_{t=0}=1$. В частности, полагая в полученном выше соотношении $t=0$, приходим к равенству

$$
F^{\prime}(z) v(z)=v^{\prime}(q) F(z)
$$

которое связывает функцию Кёнигса $F$ однопараметрической полугруппы $t \mapsto f^{t}$ в $\mathfrak{P}[q], q \in \mathbb{D}$, и ее инфинитезимальную образующую $v$.

ДокАЗАтЕЛЬСтво теоремы 2. Необходимость. Пусть $t \mapsto f^{t}-$ нетривиальная однопараметрическая полугруппа в $\mathfrak{P}[q], q \in \mathbb{D}$, и $F$ - ее функция Кёнигса. Нам нужно показать, что $F$ допускает представление (4) с некоторым $\sigma=e^{i \theta}$, $-\pi / 2<\theta<\pi / 2$, и вероятностной мерой $\mu$ на $\mathbb{T}$.

Поскольку $F$ однолистна в единичном круге $\mathbb{D}$ и $F(q)=0, F^{\prime}(q)=1$, то $F(z) /(z-q)$ не обращается в нуль в $\mathbb{D}$ и можно выделить однозначную ветвь логарифма $\ln (F(z) /(z-q))$, обращающуюся в нуль при $z=q$. При этом если $v$ - инфинитезимальная образующая однопараметрической полугруппы $t \mapsto f^{t}$, то в силу равенства (13) получаем

$$
\frac{d}{d z} \ln \frac{F(z)}{z-q}=\frac{1}{z-q}\left(\frac{(z-q) v^{\prime}(q)}{v(z)}-1\right) .
$$

Согласно формуле Берксона-Порты инфинитезимальную образующую $v$ можно представить в виде

$$
v(z)=\frac{(q-z)(1-\bar{q} z)}{p(z)},
$$

где $p$ - голоморфная в $\mathbb{D}$ функция с положительной вещественной частью. Но тогда

$$
v^{\prime}(q)=-\frac{1-|q|^{2}}{p(q)}, \quad \frac{(z-q) v^{\prime}(q)}{v(z)}-1=\frac{\left(1-|q|^{2}\right) p(z)-p(q)(1-\bar{q} z)}{p(q)(1-\bar{q} z)} .
$$

Следовательно,

$$
\frac{d}{d z} \ln \frac{F(z)}{z-q}=\frac{\left(1-|q|^{2}\right) p(z)-p(q)(1-\bar{q} z)}{p(q)(z-q)(1-\bar{q} z)} .
$$


Используя формулу Рисса-Герглотца, функцию $p$ можно представить в виде

$$
p(z)=\lambda \int_{\mathbb{T}} \frac{1+\varkappa z}{1-\varkappa z} d \nu(\varkappa)+i \beta,
$$

где $\lambda>0, \beta \in \mathbb{R}$, а $\nu$ - вероятностная мера на $\mathbb{T}$. При этом

$$
\begin{aligned}
& \left(1-|q|^{2}\right) p(z)-(1-\bar{q} z) p(q) \\
& \quad=\left(1-|q|^{2}\right)\left[\lambda \int_{\mathbb{T}} \frac{1+\varkappa z}{1-\varkappa z} d \nu(\varkappa)+i \beta\right]-(1-\bar{q} z)\left[\lambda \int_{\mathbb{T}} \frac{1+\varkappa q}{1-\varkappa q} d \nu(\varkappa)+i \beta\right] \\
& \quad=\lambda(z-q) \int_{\mathbb{T}} \frac{\varkappa(1+\varkappa q)(1-\bar{q} z)+(\varkappa+\bar{q})(1-\varkappa q)}{(1-\varkappa q)(1-\varkappa z)} d \nu(\varkappa)+i \beta \bar{q}(z-q) .
\end{aligned}
$$

Теперь дифференциальное соотношение для функции Кёнигса можно преобразовать следующим образом:

$$
\begin{aligned}
\frac{d}{d z} & \ln \frac{F(z)}{z-q} \\
& =\frac{\lambda}{p(q)} \int_{\mathbb{T}}\left(\frac{1+\varkappa q}{1-\varkappa q} \frac{\varkappa}{1-\varkappa z}+\frac{\varkappa+\bar{q}}{(1-\varkappa z)(1-\bar{q} z)}\right) d \nu(\varkappa)+\frac{i \beta}{p(q)} \frac{\bar{q}}{1-\bar{q} z} \\
& =\frac{\lambda}{p(q)} \int_{\mathbb{T}}\left(\frac{1+\varkappa q}{1-\varkappa q} \frac{\varkappa}{1-\varkappa z}+\frac{\varkappa+\bar{q}}{\varkappa-\bar{q}}\left(\frac{\varkappa}{1-\varkappa z}-\frac{\bar{q}}{1-\bar{q} z}\right)\right) d \nu(\varkappa)+\frac{i \beta}{p(q)} \frac{\bar{q}}{1-\bar{q} z} \\
& =\frac{\lambda}{p(q)} \int_{\mathbb{T}} \frac{\varkappa}{1-\varkappa z} 2 \operatorname{Re}\left\{\frac{1+\varkappa q}{1-\varkappa q}\right\} d \nu(\varkappa)-\frac{1}{p(q)} \frac{\bar{q}}{1-\bar{q} z}\left(\lambda \int_{\mathbb{T}} \frac{1+\bar{\varkappa} \bar{q}}{1-\bar{\varkappa}} d \nu(\varkappa)-i \beta\right) \\
& =\frac{2 \lambda}{p(q)} \int_{\mathbb{T}} \frac{\varkappa}{1-\varkappa z} \operatorname{Re}\left\{\frac{1+\varkappa q}{1-\varkappa q}\right\} d \nu(\varkappa)-\frac{\bar{p}(q)}{p(q)} \frac{\bar{q}}{1-\bar{q} z} .
\end{aligned}
$$

Введем в рассмотрение меру $\mu$ на $\mathbb{T}$ посредством равенства

$$
d \mu(\varkappa)=\frac{\lambda}{\operatorname{Re} p(q)} \operatorname{Re}\left\{\frac{1+\varkappa q}{1-\varkappa q}\right\} d \nu(\varkappa) .
$$

Поскольку

$$
\int_{\mathbb{T}} d \mu(\varkappa)=\frac{1}{\operatorname{Re} p(q)} \operatorname{Re}\left\{\lambda \int_{\mathbb{T}} \frac{1+\varkappa q}{1-\varkappa q} d \nu(\varkappa)\right\}=1,
$$

то $\mu$ является вероятностной мерой, и теперь

$$
\frac{d}{d z} \ln \frac{F(z)}{z-q}=\frac{2 \operatorname{Re} p(q)}{p(q)} \int_{\mathbb{T}} \frac{\varkappa}{1-\varkappa z} d \mu(\varkappa)-\frac{\overline{p(q)}}{p(q)} \frac{\bar{q}}{1-\bar{q} z} .
$$

Замечая, что $\operatorname{Re} p(q)>0$, определим $\sigma=e^{i \theta},-\pi / 2<\theta<\pi / 2$, посредством равенства $\sigma^{2}=\overline{p(q)} / p(q)$. Тогда

$$
\frac{d}{d z} \ln \frac{F(z)}{z-q}=\left(1+\sigma^{2}\right) \int_{\mathbb{T}} \frac{\varkappa}{1-\varkappa z} d \mu(\varkappa)-\sigma^{2} \frac{\bar{q}}{1-\bar{q} z}
$$

и после интегрирования по $z$ с учетом выбора ветви логарифма получаем

$$
\ln \frac{F(z)}{z-q}=\left(1+\sigma^{2}\right) \int_{\mathbb{T}} \ln \frac{1-\varkappa q}{1-\varkappa z} d \mu(\varkappa)+\sigma^{2} \ln \frac{1-\bar{q} z}{1-|q|^{2}},
$$


где под логарифмами понимаются непрерывные ветви, обращающиеся в нуль при $z=q$. Потенцируя полученное равенство, приходим к формуле (4) для функции Кёнигса, и необходимость доказана.

Достаточность. Покажем теперь, что при любом $\sigma=e^{i \theta},-\pi / 2<\theta<\pi / 2$, и любой вероятностной мере $\mu$ на $\mathbb{T}$ формула (4) определяет функцию Кёнигса некоторой однопараметрической полугруппы $t \mapsto f^{t}$ в $\mathfrak{P}[q], q \in \mathbb{D}$. Действительно, дифференцируя равенство (4), получаем

$$
\begin{aligned}
\frac{F^{\prime}(z)}{F(z)} & =\frac{1}{z-q}-\sigma^{2} \frac{\bar{q}}{1-\bar{q} z}+\left(1+\sigma^{2}\right) \int_{\mathbb{T}} \frac{\varkappa}{1-\varkappa z} d \mu(\varkappa) \\
& =\frac{1}{z-q}-\sigma^{2} \frac{\bar{q}}{1-\bar{q} z}+\left(1+\sigma^{2}\right) \int_{\mathbb{T}}\left(\frac{\varkappa-\bar{q}}{(1-\bar{q} z)(1-\varkappa z)}+\frac{\bar{q}}{1-\bar{q} z}\right) d \mu(\varkappa) \\
& =\frac{1-|q|^{2}}{(z-q)(1-\bar{q} z)}+\left(1+\sigma^{2}\right) \int_{\mathbb{T}} \frac{\varkappa-\bar{q}}{(1-\bar{q} z)(1-\varkappa z)} d \mu(\varkappa) .
\end{aligned}
$$

Следовательно,

$$
\begin{aligned}
\bar{\sigma}(z-q)(1-\bar{q} z) \frac{F^{\prime}(z)}{F(z)} \\
\quad=\left(1-|q|^{2}\right)\left[\bar{\sigma}+(\sigma+\bar{\sigma}) \int_{\mathbb{T}} \frac{(z-q)(\varkappa-\bar{q})}{\left(1-|q|^{2}\right)(1-\varkappa z)} d \mu(\varkappa)\right]=\left(1-|q|^{2}\right) p(z),
\end{aligned}
$$

где

$$
\begin{aligned}
p(z) & =\bar{\sigma}+(\sigma+\bar{\sigma}) \int_{\mathbb{T}} \frac{(z-q)(\varkappa-\bar{q})}{\left(1-|q|^{2}\right)(1-\varkappa z)} d \mu(\varkappa) \\
& =\bar{\sigma}-\frac{\sigma+\bar{\sigma}}{2}+\frac{\sigma+\bar{\sigma}}{2} \int_{\mathbb{T}} \frac{1+\varkappa z}{1-\varkappa z} \frac{|1-\varkappa q|^{2}}{1-|q|^{2}} d \mu(\varkappa)-i 2(\operatorname{Re} \sigma) \int_{\mathbb{T}} \frac{\operatorname{Im}\{\varkappa q\}}{1-|q|^{2}} d \mu(\varkappa) \\
& =(\operatorname{Re} \sigma) \int_{\mathbb{T}} \frac{1+\varkappa z}{1-\varkappa z} \frac{|1-\varkappa q|^{2}}{1-|q|^{2}} d \mu(\varkappa)-i \operatorname{Im}\left\{\sigma+2(\operatorname{Re} \sigma) \int_{\mathbb{T}} \frac{\varkappa q}{1-|q|^{2}} d \mu(\varkappa)\right\} .
\end{aligned}
$$

Из последнего равенства видно, что $\operatorname{Re} p(z)>0$ при $z \in \mathbb{D}$.

Покажем теперь, что $F$ однолистна в единичном круге $\mathbb{D}$ и отображает его на $\theta$-спиральную область, т.е. $F(\mathbb{D})$ вместе с каждой точкой $F(z), z \in \mathbb{D}$, содержит полную дугу $\theta$-спирали $w(t)=F(z) e^{-\sigma t}, t \geqslant 0$. Напомним (см., например, [13], [14]), что аналитическая в $\mathbb{D}$ функция $\Phi$, удовлетворяющая условиям $\Phi(0)=0, \Phi^{\prime}(0) \neq 0$ и $\Phi(\zeta) \neq 0$ при $0<|\zeta|<1$, является $\theta$-спиральной в том и только том случае, если

$$
\operatorname{Re}\left\{\bar{\sigma} \frac{\zeta \Phi^{\prime}(\zeta)}{\Phi(\zeta)}\right\}>0
$$

при $\zeta \in \mathbb{D}$.

Пусть

$$
\zeta=L(z)=\frac{q-z}{1-\bar{q} z}
$$

и $\Phi(\zeta)=F\left(L^{-1}(\zeta)\right)$. Тогда функция $\Phi$ будет удовлетворять условиям $\Phi(0)=$ $F(q)=0, \Phi^{\prime}(0) \neq 0$ и $\Phi(\zeta) \neq 0$ при $0<|\zeta|<1$. Кроме того,

$$
\frac{\zeta \Phi^{\prime}(\zeta)}{\Phi(\zeta)}=\frac{L(z) F^{\prime}(z)}{L^{\prime}(z) F(z)}=\frac{(z-q)(1-\bar{q} z)}{1-|q|^{2}} \frac{F^{\prime}(z)}{F(z)}
$$


и, следовательно,

$$
\operatorname{Re}\left\{\bar{\sigma} \frac{\zeta \Phi^{\prime}(\zeta)}{\Phi(\zeta)}\right\}=\operatorname{Re} p(z)>0
$$

при $\zeta \in \mathbb{D}$. Таким образом, $F(\mathbb{D})=\Phi(\mathbb{D})$ является $\theta$-спиральной областью, и мы можем определить однопараметрическую полугруппу $t \mapsto f^{t}$ в $\mathfrak{P}$ посредством равенств

$$
f^{t}(z)=F^{-1}\left(e^{-\sigma t} F(z)\right)
$$

где $t \geqslant 0$. Поскольку $F(q)=0$, то $q$ будет неподвижной точкой для всех функций $f^{t}, t>0$. Кроме того, инфинитезимальная образующая $v$ однопараметрической полугруппы $t \mapsto f^{t}$ будет иметь вид

$$
v(z)=\left.\frac{\partial}{\partial t} F^{-1}\left(e^{-\sigma t} F(z)\right)\right|_{t=0}=-\sigma \frac{F(z)}{F^{\prime}(z)}=\frac{(q-z)(1-\bar{q} z)}{\left(1-|q|^{2}\right) p(z)} .
$$

Таким образом, $t \mapsto f^{t}$ является однопараметрической полугруппой в $\mathfrak{P}[q]$, а $F$ является ее функцией Кёнигса, и теорема 2 доказана.

ДокАЗАТЕЛЬство теоремы 3. Пусть теперь $t \mapsto f^{t}$ - однопараметрическая полугруппа в $\mathfrak{P}[q ; a]$, т.е. функции $f^{t}, t>0$, кроме точки Данжуа-Вольфа $q \in \mathbb{D}$ оставляют неподвижной точку $a \in \mathbb{T}$, в которой они имеют конечные угловые производные. Тогда в силу теоремы 1 и леммы 2 инфинитезимальную образующую $v$ этой однопараметрической полугруппы можно представить в виде

$$
v(z)=\frac{(q-z)(1-\bar{q} z)}{\alpha\left(\frac{1+\bar{a} z}{1-\bar{a} z}+g(z)\right)}
$$

где $\alpha>0$, а функция $g$ голоморфна в $\mathbb{D}$ и имеет неотрицательную вещественную часть. Случай, когда функция $g$ тождественно равна мнимой константе, тривиален. Поэтому будем считать, что $\operatorname{Re} g(z)>0$ при $z \in \mathbb{D}$. Другими словами, в общем представлении (рассмотренном выше) инфинитезимальной образующей

$$
v(z)=\frac{(q-z)(1-\bar{q} z)}{p(z)}
$$

функция $p$ имеет специальный вид

$$
p(z)=\alpha\left(\frac{1+\bar{a} z}{1-\bar{a} z}+g(z)\right) .
$$

По теореме Рисса-Герглотца функцию $g$ можно представить в виде

$$
g(z)=\lambda \int_{\mathbb{T}} \frac{1+\varkappa z}{1-\varkappa z} d \nu(\varkappa)+i \beta,
$$

где $\lambda>0, \beta \in \mathbb{R}$, а $\nu$ - вероятностная мера на $\mathbb{T}$. Но тогда

$$
\begin{aligned}
& \left(1-|q|^{2}\right) p(z)-(1-\bar{q} z) p(q)=\alpha\left[\left(1-|q|^{2}\right) \frac{1+\bar{a} z}{1-\bar{a} z}-(1-\bar{q} z) \frac{1+\bar{a} q}{1-\bar{a} q}\right. \\
& \left.+\lambda \int_{\mathbb{T}}\left(\left(1-|q|^{2}\right) \frac{1+\varkappa z}{1-\varkappa z}-(1-\bar{q} z) \frac{1+\varkappa q}{1-\varkappa q}\right) d \nu(\varkappa)+i \beta \bar{q}(z-q)\right]
\end{aligned}
$$




$$
\begin{aligned}
=\alpha & (z-q)\left[\frac{\bar{a}(1+\bar{a} q)(1-\bar{q} z)+(\bar{a}+\bar{q})(1-\bar{a} q)}{(1-\bar{a} q)(1-\bar{a} z)}\right. \\
& \left.+\lambda \int_{\mathbb{T}} \frac{\varkappa(1+\varkappa q)(1-\bar{q} z)+(\varkappa+\bar{q})(1-\varkappa q)}{(1-\varkappa q)(1-\varkappa z)} d \nu(\varkappa)+i \beta \bar{q}\right],
\end{aligned}
$$

и для функции Кёнигса $F$ получаем следующее дифференциальное соотношение:

$$
\begin{aligned}
\frac{d}{d z} \ln \frac{F(z)}{z-q}=\frac{\alpha}{p(q)}\left[\frac{1+\bar{a} q}{1-\bar{a} q} \frac{\bar{a}}{1-\bar{a} z}+\frac{\bar{a}+\bar{q}}{(1-\bar{a} z)(1-\bar{q} z)}\right. \\
\left.+\lambda \int_{\mathbb{T}}\left(\frac{1+\varkappa q}{1-\varkappa q} \frac{\varkappa}{1-\varkappa z}+\frac{\varkappa+\bar{q}}{(1-\varkappa z)(1-\bar{q} z)}\right) d \nu(\varkappa)+i \beta \frac{\bar{q}}{1-\bar{q} z}\right] \\
=\frac{\alpha}{p(q)}\left[\frac{2 \bar{a}}{1-\bar{a} z} \operatorname{Re}\left\{\frac{1+\bar{a} q}{1-\bar{a} q}\right\}-\frac{\bar{q}}{1-\bar{q} z} \frac{1+a \bar{q}}{1-a \bar{q}}\right. \\
\left.\quad+\lambda \int_{\mathbb{T}} \frac{2 \varkappa}{1-\varkappa z} \operatorname{Re}\left\{\frac{1+\varkappa q}{1-\varkappa q}\right\} d \nu(\varkappa)-\lambda \frac{\bar{q}}{1-\bar{q} z} \int_{\mathbb{T}} \frac{1+\overline{\varkappa q}}{1-\overline{\varkappa q}} d \nu(\varkappa)+i \beta \frac{\bar{q}}{1-\bar{q} z}\right] \\
=\frac{2 \alpha}{p(q)}\left[\frac{\bar{a}}{1-\bar{a} z} \operatorname{Re}\left\{\frac{1+\bar{a} q}{1-\bar{a} q}\right\}+\lambda \int_{\mathbb{T}} \frac{\varkappa}{1-\varkappa z} \operatorname{Re}\left\{\frac{1+\varkappa q}{1-\varkappa q}\right\} d \nu(\varkappa)\right]-\frac{\overline{p(q)}}{p(q)} \frac{\bar{q}}{1-\bar{q} z} .
\end{aligned}
$$

Обозначим

$$
\delta=\int_{\mathbb{T}} \operatorname{Re}\left\{\frac{1+\varkappa q}{1-\varkappa q}\right\} d \nu(\varkappa)
$$

и определим вероятностную на $\mathbb{T}$ меру $\mu$ посредством равенства

$$
d \mu(\varkappa)=\frac{1}{\delta} \operatorname{Re}\left\{\frac{1+\varkappa q}{1-\varkappa q}\right\} d \nu(\varkappa) .
$$

Тогда полученное соотношение перепишется в виде

$$
\frac{d}{d z} \ln \frac{F(z)}{z-q}=\frac{2 \alpha}{p(q)}\left[\frac{\bar{a}}{1-\bar{a} z} \operatorname{Re}\left\{\frac{1+\bar{a} q}{1-\bar{a} q}\right\}+\lambda \delta \int_{\mathbb{T}} \frac{\varkappa}{1-\varkappa z} d \mu(\varkappa)\right]-\frac{\overline{p(q)}}{p(q)} \frac{\bar{q}}{1-\bar{q} z} .
$$

Полагая теперь

$$
\lambda_{1}=\frac{\alpha}{\operatorname{Re} p(q)} \operatorname{Re}\left\{\frac{1+\bar{a} q}{1-\bar{a} q}\right\}, \quad \lambda_{2}=\frac{\alpha \lambda \delta}{\operatorname{Re} p(q)},
$$

получим положительные числа, удовлетворяющие условию

$$
\lambda_{1}+\lambda_{2}=\frac{\alpha}{\operatorname{Re} p(q)} \operatorname{Re}\left\{\frac{1+\bar{a} q}{1-\bar{a} q}+\lambda \int_{\mathbb{T}} \frac{1+\varkappa q}{1-\varkappa q} d \nu(\varkappa)\right\}=1 .
$$

В этих терминах дифференциальное соотношение для функции Кёнигса $F$ принимает вид

$$
\frac{d}{d z} \ln \frac{F(z)}{z-q}=\frac{2 \operatorname{Re} p(q)}{p(q)}\left[\lambda_{1} \frac{\bar{a}}{1-\bar{a} z}+\lambda_{2} \int_{\mathbb{T}} \frac{\varkappa}{1-\varkappa z} d \mu(\varkappa)\right]-\frac{\overline{p(q)}}{p(q)} \frac{\bar{q}}{1-\bar{q} z} .
$$


Снова определим $\sigma=e^{i \theta},-\pi / 2<\theta<\pi / 2$, посредством равенства $\sigma^{2}=$ $\overline{p(q)} / p(q)$ и перепишем соотношение для $F$ в виде

$$
\frac{d}{d z} \ln \frac{F(z)}{z-q}=\left(1+\sigma^{2}\right)\left[\lambda_{1} \frac{\bar{a}}{1-\bar{a} z}+\lambda_{2} \int_{\mathbb{T}} \frac{\varkappa}{1-\varkappa z} d \mu(\varkappa)\right]-\sigma^{2} \frac{\bar{q}}{1-\bar{q} z} .
$$

Интегрирование этого равенства по $z$ дает

$$
\ln \frac{F(z)}{z-q}=\sigma^{2} \ln \frac{1-\bar{q} z}{1-|q|^{2}}+\left(1+\sigma^{2}\right)\left[\lambda_{1} \ln \frac{1-\bar{a} q}{1-\bar{a} z}+\lambda_{2} \int_{\mathbb{T}} \ln \frac{1-\varkappa q}{1-\varkappa z} d \mu(\varkappa)\right],
$$

где под логарифмами понимаются непрерывные ветви, обращающиеся в нуль при $z=q$. Потенцирование последнего равенства приводит к виду функции Кёнигса $F$, который определяет формула (5).

Обратно, пусть функция $F$ имеет вид (5) с некоторыми $\lambda_{1}>0, \lambda_{2} \geqslant 0$, $\lambda_{1}+\lambda_{2}=1, \sigma=e^{i \theta},-\pi / 2<\theta<\pi / 2$, и вероятностной мерой $\mu$ на $\mathbb{T}$. Покажем, что найдется такая однопараметрическая полугруппа $t \mapsto f^{t}$ в $\mathfrak{P}[q ; a], q \in \mathbb{D}$, $a \in \mathbb{T}$, что $F$ является для нее функцией Кёнигса.

Выполняя очевидные преобразования, получаем

$$
\begin{aligned}
\frac{F^{\prime}(z)}{F(z)=} & \frac{1}{z-q}-\sigma^{2} \frac{\bar{q}}{1-\bar{q} z}+\lambda_{1}\left(1+\sigma^{2}\right) \frac{\bar{a}}{1-\bar{a} z}+\lambda_{2}\left(1+\sigma^{2}\right) \int_{\mathbb{T}} \frac{\varkappa}{1-\varkappa z} d \mu(\varkappa) \\
= & \frac{1}{z-q}+\frac{\bar{q}}{1-\bar{q} z} \\
& \quad+\left(1+\sigma^{2}\right)\left[\lambda_{1}\left(\frac{\bar{a}}{1-\bar{a} z}-\frac{\bar{q}}{1-\bar{q} z}\right)+\lambda_{2} \int_{\mathbb{T}}\left(\frac{\varkappa}{1-\varkappa z}-\frac{\bar{q}}{1-\bar{q} z}\right) d \mu(\varkappa)\right] \\
= & \frac{1-|q|^{2}}{(z-q)(1-\bar{q} z)} \\
& \quad+\left(1+\sigma^{2}\right)\left[\lambda_{1} \frac{\bar{a}-\bar{q}}{(1-\bar{a} z)(1-\bar{q} z)}+\frac{\lambda_{2}}{1-\bar{q} z} \int_{\mathbb{T}} \frac{\varkappa-\bar{q}}{1-\varkappa z} d \mu(\varkappa)\right]
\end{aligned}
$$

и, далее,

$$
\begin{aligned}
& \bar{\sigma}(z-q)(1-\bar{q} z) \frac{F^{\prime}(z)}{F(z)} \\
& \quad=\bar{\sigma}\left(1-|q|^{2}\right)+(\sigma+\bar{\sigma})\left[\lambda_{1} \frac{(\bar{a}-\bar{q})(z-q)}{1-\bar{a} z}+\lambda_{2} \int_{\mathbb{T}} \frac{(\varkappa-\bar{q})(z-q)}{1-\varkappa z} d \mu(\varkappa)\right] .
\end{aligned}
$$

Замечая, что

$$
\begin{aligned}
& \frac{(\bar{a}-\bar{q})(z-q)}{1-\bar{a} z}=\frac{|1-\bar{a} q|^{2}}{2} \frac{1+\bar{a} z}{1-\bar{a} z}-\frac{1}{2}\left(1-|q|^{2}\right)-i \operatorname{Im}\{\bar{a} q\} \\
& \frac{(\varkappa-\bar{q})(z-q)}{1-\varkappa z}=\frac{|1-\varkappa|^{2}}{2} \frac{1+\varkappa z}{1-\varkappa z}-\frac{1}{2}\left(1-|q|^{2}\right)-i \operatorname{Im}\{\varkappa q\}
\end{aligned}
$$

получаем

$$
\begin{gathered}
\bar{\sigma}(z-q)(1-\bar{q} z) \frac{F^{\prime}(z)}{F(z)}=(\operatorname{Re} \sigma)\left[\lambda_{1}|1-\bar{a} q|^{2} \frac{1+\bar{a} z}{1-\bar{a} z}+\lambda_{2} \int_{\mathbb{T}} \frac{1+\varkappa z}{1-\varkappa z}|1-\varkappa q|^{2} d \mu(\varkappa)\right] \\
-i \operatorname{Im}\left\{\left(1-|q|^{2}\right) \sigma+2 q(\operatorname{Re} \sigma)\left(\lambda_{1} \bar{a}+\lambda_{2} \int_{\mathbb{T}} \varkappa d \mu(\varkappa)\right)\right\} .
\end{gathered}
$$


Поскольку в правой части последнего равенства стоит функция с положительной вещественной частью, то $F(\mathbb{D})$ является $\theta$-спиральной областью, и снова можно определить однопараметрическую полугруппу $t \mapsto f^{t}$ в $\mathfrak{P}[q]$ посредством равенства

$$
f^{t}(z)=F^{-1}\left(e^{-\sigma t} F(z)\right)
$$

где $t \geqslant 0$. Инфинитезимальная образующая $v$ однопараметрической полугруппы $t \mapsto f^{t}$ в терминах функции $F$ записывается в виде

$$
v(z)=-\sigma \frac{F(z)}{F^{\prime}(z)}=-\frac{F(z)}{\bar{\sigma} F^{\prime}(z)} .
$$

Однако из предыдущих вычислений выводим

$$
(z-q)(1-\bar{q} z) \frac{\bar{\sigma} F^{\prime}(z)}{F(z)}=\alpha\left(\frac{1+\bar{a} z}{1-\bar{a} z}+g(z)\right),
$$

где $\alpha>0$, а $g$ - голоморфная в $\mathbb{D}$ функция с неотрицательной вещественной частью. Следовательно,

$$
v(z)=\frac{(q-z)(1-\bar{q} z)}{\alpha\left(\frac{1+\bar{a} z}{1-\bar{a} z}+g(z)\right)},
$$

что в силу леммы 2 эквивалентно представлению из теоремы 1. Это означает, что $t \mapsto f^{t}$ является однопараметрической полугруппой в $\mathfrak{P}[q ; a]$, а $F$ является ее функцией Кёнигса. Таким образом, теорема 3 доказана.

\section{§6. Функция Кёнигса в случае граничной точки Данжуа-Вольфа}

В случае, когда точка Данжуа-Вольфа $q$ функции $f \in \mathfrak{P}$ является внутренней (т.е. $q \in \mathbb{D})$, происхождение функции Кёнигса обусловлено, с одной стороны, асимптотическим поведением итераций функции $f$ и связью с решением функционального уравнения - с другой.

Пусть теперь $f \in \mathfrak{P}[q], q \in \mathbb{T}$, т.е. точка Данжуа-Вольфа $q$ является граничной. В монографии [6] показано, что если угловая производная $f^{\prime}(q)$ не равна 1, то можно нормировать последовательность натуральных итераций $f^{n}$, $n=1,2, \ldots$, так, чтобы она сходилась к некоторой аналитической функции, которую Валирон также называет функцией Кёнигса. Эта функция, как и в случае внутренней точки Данжуа-Вольфа, является общим решением для всех итераций функции $f$ уравнения Шрёдера. Случай $f^{\prime}(q)=1$ оказался значительно сложнее. В работах [15], [16] изучались другие способы нормировки последовательности итераций - так, чтобы она сходилась к непостоянной аналитической функции. При этом во всех выделенных случаях, кроме гиперболического, предельная функция является решением функционального уравнения Абеля для всех итераций функции $f$. Уравнение Абеля возникает также при изучении итераций вероятностных производящих функций, относящихся к случаю критических ветвящихся процессов (см. [17]). Для однопараметрической полугруппы $t \mapsto f^{t}$ в $\mathfrak{P}[q]$ при $q \in \mathbb{T}$ единственным образом возникает функция $F$, которая для всех $f^{t}, t>0$, удовлетворяет функциональному уравнению Абеля

$$
F \circ f^{t}(z)=F(z)+t .
$$


Действительно, пусть $t \mapsto f^{t}$ - однопараметрическая полугруппа в $\mathfrak{P}[q]$, $q \in \mathbb{T}$, и $v$ - ее инфинитезимальная образующая. Поскольку $|q|=1$, то инфинитезимальную образующую $v$ можно в силу формулы Берксона-Порты представить в виде

$$
v(z)=\frac{q(1-\bar{q} z)^{2}}{p(z)},
$$

где $p$ - голоморфная с неотрицательной вещественной частью в $\mathbb{D}$ функция. Случай, когда $p$ тождественно равняется мнимой константе, отвечает однопараметрической полугруппе дробно-линейных преобразований единичного круга на себя. Поэтому в дальнейшем будем предполагать, что $\operatorname{Re} p(z)>0$ при $z \in \mathbb{D}$. Определим голоморфную в $\mathbb{D}$ функцию $F$ посредством равенств $F(0)=0$ и

$$
F^{\prime}(z)=\frac{p(z)}{q(1-\bar{q} z)^{2}} .
$$

Это определение корректно, поскольку $\mathbb{D}$ - односвязная область. Кроме того, для функции $L(z)=z /(q-z)$, которая отображает $\mathbb{D}$ на полуплоскость, выполняется равенство

$$
\frac{F^{\prime}(z)}{L^{\prime}(z)}=p(z) .
$$

Отсюда следует, что $F$ является близкой к выпуклой функцией (см., например, [14]). В частности, $F$ однолистна в $\mathbb{D}$. Кроме того, образ $F(\mathbb{D})$ единичного круга обладает тем свойством, что вместе с каждой точкой $w \in F(\mathbb{D})$ он содержит и весь луч $\{w+t: t \geqslant 0\}$. Действительно, пусть $w_{0} \in F(\mathbb{D})$, т.е. $w_{0}=F\left(z_{0}\right)$ при некотором $z_{0} \in \mathbb{D}$. Тогда кривая $w(t)=F\left(f^{t}\left(z_{0}\right)\right), t \geqslant 0$, содержится в $F(\mathbb{D})$ и $w(0)=w_{0}$. При этом

$$
\frac{d}{d t} w(t)=F^{\prime}\left(f^{t}\left(z_{0}\right)\right) v\left(f^{t}\left(z_{0}\right)\right)=1,
$$

т.е. $w(t)=w_{0}+t, t \geqslant 0$. Таким образом, при всех $t \geqslant 0$ выполняется равенство $F\left(f^{t}(z)\right)=F(z)+t, z \in \mathbb{D}$.

Итак, с каждой однопараметрической полугруппой $t \mapsto f^{t}$ в $\mathfrak{P}[q], q \in \mathbb{T}$, однозначно ассоциируется функция $F$, которая является решением уравнения (14) при всех $t \geqslant 0$ и удовлетворяет условию $F(0)=0$. Эту функцию мы будем называть функиией Кёнигса однопараметрической полугруппь $t \mapsto f^{t}$. Как следует из геометрических свойств функции $F$, однопараметрическая полугруппа $t \mapsto f^{t}$ восстанавливается с помощью своей функции Кёнигса посредством равенства

$$
f^{t}(z)=F^{-1}(F(z)+t)
$$

где $t \geqslant 0$.

Приведем теперь параметрическое представление классов функций Кёнигса, отвечающих однопараметрическим полугруппам в $\mathfrak{P}[q]$ и $\mathfrak{P}[q ; a], q \in \mathbb{T}, a \in \mathbb{T}$.

ДоказАтельство теоремы 4. Допустим вначале, что $t \mapsto f^{t}$ - однопараметрическая полугруппа в $\mathfrak{P}[q], q \in \mathbb{T}$. Тогда ее инфинитезимальная образующая $v$ имеет вид (15), а функция Кёнигса определяется равенством (16). 
Представим функцию $p$ в виде

$$
p(z)=\alpha \int_{\mathbb{T}} \frac{1+\varkappa z}{1-\varkappa z} d \nu(\varkappa)+i \beta
$$

где $\alpha>0, \beta \in \mathbb{R}$, а $\nu$ - вероятностная мера на $\mathbb{T}$. Тогда равенство (16) переписывается следующим образом:

$$
\begin{aligned}
F^{\prime}(z)= & \frac{\bar{q}}{(1-\bar{q} z)^{2}}\left[\alpha \int_{\mathbb{T}} \frac{1+\varkappa z}{1-\varkappa z} d \nu(\varkappa)+i \beta\right] \\
= & i \beta \frac{\bar{q}}{(1-\bar{q} z)^{2}}+\alpha \nu(\{\bar{q}\}) \frac{\bar{q}(1+\bar{q} z)}{(1-\bar{q} z)^{3}}+\alpha \int_{\mathbb{T} \backslash\{\bar{q}\}}\left(\frac{1+\varkappa q}{1-\varkappa q} \frac{\bar{q}}{(1-\bar{q} z)^{2}}\right. \\
& \left.-\frac{2 \varkappa q}{(1-\varkappa q)^{2}} \frac{\bar{q}}{1-\bar{q} z}+\frac{2 \varkappa q}{(1-\varkappa q)^{2}} \frac{\varkappa}{1-\varkappa z}\right) d \nu(\varkappa) .
\end{aligned}
$$

Интегрирование этого равенства с учетом условия $F(0)=0$ дает

$$
\begin{aligned}
F(z)=i \beta & \frac{\bar{q} z}{1-\bar{q} z}+\alpha \nu(\{\bar{q}\}) \frac{\bar{q} z}{(1-\bar{q} z)^{2}} \\
& +\alpha \int_{\mathbb{T} \backslash\{\bar{q}\}}\left(\frac{1+\varkappa q}{1-\varkappa q} \frac{\bar{q} z}{1-\bar{q} z}-\frac{2 \varkappa q}{(1-\varkappa q)^{2}} \ln \frac{1-\varkappa z}{1-\bar{q} z}\right) d \nu(\varkappa),
\end{aligned}
$$

где под логарифмом понимается непрерывная ветвь, обращающаяся в нуль при $z=0$. Замечая, что

$$
\frac{1+\varkappa q}{1-\varkappa q}=\frac{i \operatorname{Im}\{\varkappa q\}}{1-\operatorname{Re}\{\varkappa q\}}, \quad-\frac{2 \varkappa q}{(1-\varkappa q)^{2}}=\frac{1}{1-\operatorname{Re}\{\varkappa q\}},
$$

получаем

$$
\begin{aligned}
F(z)= & i \beta \frac{\bar{q} z}{1-\bar{q} z}+\alpha \nu(\{\bar{q}\}) \frac{\bar{q} z}{(1-\bar{q} z)^{2}} \\
& +\alpha \int_{\mathbb{T} \backslash\{\bar{q}\}}\left(\ln \frac{1-\varkappa z}{1-\bar{q} z}+i(\operatorname{Im}\{\varkappa q\}) \frac{\bar{q} z}{1-\bar{q} z}\right) \frac{d \nu(\varkappa)}{1-\operatorname{Re}\{\varkappa q\}} \\
= & i \beta \frac{\bar{q} z}{1-\bar{q} z}+\lambda_{1} \frac{\bar{q} z}{(1-\bar{q} z)^{2}} \\
& +\lambda_{2} \int_{\mathbb{T} \backslash\{\bar{q}\}}\left(\ln \frac{1-\varkappa z}{1-\bar{q} z}+i(\operatorname{Im}\{\varkappa q\}) \frac{\bar{q} z}{1-\bar{q} z}\right) \frac{d \mu(\varkappa)}{1-\operatorname{Re}\{\varkappa q\}},
\end{aligned}
$$

где $\beta \in \mathbb{R}, \lambda_{1}, \lambda_{2} \geqslant 0$, а $\mu$ - вероятностная мера на $\mathbb{T} \backslash\{\bar{q}\}$. Сходимость интеграла в последнем равенстве обеспечивается тем, что

$$
\lim _{\varkappa \rightarrow \bar{q}}\left\{\frac{1}{1-\operatorname{Re}\{\varkappa q\}}\left(\ln \frac{1-\varkappa z}{1-\bar{q} z}+i(\operatorname{Im}\{\varkappa q\}) \frac{\bar{q} z}{1-\bar{q} z}\right)\right\}=\frac{\bar{q} z}{(1-\bar{q} z)^{2}}
$$


Обратно, пусть для некоторых $\lambda_{1}, \lambda_{2} \geqslant 0, \beta \in \mathbb{R}$ и вероятностной меры $\mu$ на $\mathbb{T} \backslash\{\bar{q}\}$ определена функция $F$ по формуле (6). Тогда

$$
\begin{aligned}
F^{\prime}(z)= & i \beta \frac{\bar{q}}{(1-\bar{q} z)^{2}}+\lambda_{1} \frac{\bar{q}(1+\bar{q} z)}{(1-\bar{q} z)^{3}} \\
& \quad+\lambda_{2} \int_{\mathbb{T} \backslash\{\bar{q}\}}\left(\frac{\bar{q}-\varkappa}{(1-\bar{q} z)(1-\varkappa z)}+i(\operatorname{Im}\{\varkappa q\}) \frac{\bar{q}}{(1-\bar{q} z)^{2}}\right) \frac{d \mu(\varkappa)}{1-\operatorname{Re}\{\varkappa q\}} \\
= & i \beta \frac{\bar{q}}{(1-\bar{q} z)^{2}}+\lambda_{1} \frac{\bar{q}(1+\bar{q} z)}{(1-\bar{q} z)^{3}}+\lambda_{2} \int_{\mathbb{T} \backslash\{\bar{q}\}} \frac{\bar{q}(1+\varkappa z)}{(1-\bar{q} z)^{2}(1-\varkappa z)} d \mu(\varkappa) \\
= & \frac{p(z)}{q(1-\bar{q} z)^{2}},
\end{aligned}
$$

где

$$
p(z)=i \beta+\lambda_{1} \frac{1+\bar{q} z}{1-\bar{q} z}+\lambda_{2} \int_{\mathbb{T} \backslash\{\bar{q}\}} \frac{1+\varkappa z}{1-\varkappa z} d \mu(\varkappa) .
$$

Поскольку $\operatorname{Re} p(z) \geqslant 0$ при $z \in \mathbb{D}$, то легко проверяется, что $F$ является близкой к выпуклой функцией и, следовательно, однолистной. Для выяснения геометрии образа $F(\mathbb{D})$ единичного круга изучим свойства образов орициклов

$$
H_{\varrho}(q)=\left\{z \in \mathbb{D}: \operatorname{Re} \frac{1+\bar{q} z}{1-\bar{q} z}>\frac{1}{\varrho}\right\},
$$

где $\varrho>0$, при отображении их посредством $F$.

Пусть $z=z(s),-\infty<s<\infty,-$ параметризация границы $\partial H_{\varrho}(q)$ орицикла $H_{\varrho}(q)$ такая, что при возрастании параметра $s$ точка $z(s)$ движется по $\partial H_{\varrho}(q)$ в положительном направлении, т.е. орицикл $H_{\varrho}(q)$ остается слева. При этом

$$
\operatorname{Im} \frac{1+\bar{q} z(s)}{1-\bar{q} z(s)}
$$

будет строго монотонно убывать по $s$ и

$$
\frac{d}{d s} \operatorname{Im} \frac{1+\bar{q} z(s)}{1-\bar{q} z(s)}=\operatorname{Im} \frac{2 \bar{q} z^{\prime}(s)}{(1-\bar{q} z(s))^{2}}<0 .
$$

Кроме того, дифференцируя по $s$ равенство

$$
\operatorname{Re} \frac{1+\bar{q} z(s)}{1-\bar{q} z(s)}=\frac{1}{\varrho},
$$

получаем

$$
\operatorname{Re} \frac{\bar{q} z^{\prime}(s)}{(1-\bar{q} z(s))^{2}}=0 .
$$

Таким образом, при всех $s,-\infty<s<\infty$, выражение

$$
\frac{\bar{q} z^{\prime}(s)}{(1-\bar{q} z(s))^{2}}
$$

принимает значения на отрицательной части мнимой оси. Но тогда

$$
\frac{d}{d s} \operatorname{Im} F(z(s))=\operatorname{Im}\left\{F^{\prime}(z(s)) z^{\prime}(s)\right\}=\operatorname{Im}\left\{\frac{z^{\prime}(s) p(z(s))}{q(1-\bar{q} z(s))^{2}}\right\}<0 .
$$


Это означает, что образ $F\left(H_{\varrho}(q)\right)$ орицикла $H_{\varrho}(q)$ представляет собой область, которая вместе с каждой точкой $w$ содержит и весь луч $w+t, t \geqslant 0$. Это свойство распространяется и на весь образ $F(\mathbb{D})$ единичного круга. Следовательно, при каждом $t>0$ корректно определены функции

$$
f^{t}(z)=F^{-1}(F(z)+t) .
$$

Легко видеть, что $t \mapsto f^{t}$ является однопараметрической полугруппой в $\mathfrak{P}[q]$, а $F$ - ее функция Кёнигса. В результате теорема 4 доказана.

ДокАЗАТЕЛЬСтво теоремы 5. Пусть теперь $t \mapsto f^{t}$ - однопараметрическая полугруппа в $\mathfrak{P}[q ; a], q \in \mathbb{T}, a \in \mathbb{T}$, т.е. $q$ является точкой Данжуа-Вольфа для функций $f^{t}, t>0$, и кроме $q$ неподвижной также является точка $a \neq q$, в которой функции $f^{t}$ имеют конечные угловые производные. Поскольку $|q|=1$, то в силу теоремы 1 и леммы 2 инфинитезимальную образующую $v$ этой однопараметрической полугруппы можно представить в виде

$$
v(z)=\frac{q(1-\bar{q} z)^{2}}{\alpha\left(\frac{1+\bar{a} z}{1-\bar{a} z}+g(z)\right)},
$$

где $\alpha>0$, а функция $g$ голоморфна в $\mathbb{D}$ и имеет неотрицательную вещественную часть в $\mathbb{D}$. Равенство (16), определяющее функцию Кёнигса, запишется в этом случае в виде

$$
F^{\prime}(z)=\frac{\alpha}{q(1-\bar{q} z)^{2}}\left(\frac{1+\bar{a} z}{1-\bar{a} z}+g(z)\right) .
$$

Для функции $g$ в силу теоремы Рисса-Герглотца имеет место интегральное представление

$$
g(z)=\lambda \int_{\mathbb{T}} \frac{1+\varkappa z}{1-\varkappa z} d \nu(\varkappa)+i \beta,
$$

где $\lambda \geqslant 0, \beta \in \mathbb{R}$, а $\nu$ - вероятностная мера на $\mathbb{T}$. С учетом этого равенство, определяющее функцию Кёнигса, перепишем следующим образом:

$$
\begin{aligned}
F^{\prime}(z)= & \frac{\alpha}{q(1-\bar{q} z)^{2}}\left(\frac{1+\bar{a} z}{1-\bar{a} z}+\lambda \int_{\mathbb{T}} \frac{1+\varkappa z}{1-\varkappa z} d \nu(\varkappa)+i \beta\right) \\
= & i \alpha \beta \frac{\bar{q}}{(1-\bar{q} z)^{2}}+\alpha \frac{\bar{q}(1+\bar{a} z)}{(1-\bar{q} z)^{2}(1-\bar{a} z)}+\alpha \lambda \nu(\{\bar{q}\}) \frac{\bar{q}(1+\bar{q} z)}{(1-\bar{q} z)^{3}} \\
& \quad+\alpha \lambda \int_{\mathbb{T} \backslash\{\bar{q}\}}\left(\frac{\bar{q}-\varkappa}{(1-\bar{q} z)(1-\varkappa z)}+i(\operatorname{Im}\{\varkappa q\}) \frac{\bar{q}}{(1-\bar{q} z)^{2}}\right) \frac{d \nu(\varkappa)}{1-\operatorname{Re}\{\varkappa q\}} .
\end{aligned}
$$

После интегрирования этого равенства с учетом условия $F(0)=0$ получаем

$$
\begin{aligned}
F(z)=i & \alpha \beta \frac{\bar{q} z}{1-\bar{q} z}+\frac{\alpha}{1-\operatorname{Re}\{\bar{a} q\}} \ln \frac{1-\bar{a} z}{1-\bar{q} z} \\
& +i \frac{\alpha \operatorname{Im}\{\bar{a} q\}}{1-\operatorname{Re}\{\bar{a} q\}} \frac{\bar{q} z}{1-\bar{q} z}+\alpha \lambda \nu(\{\bar{q}\}) \frac{\bar{q} z}{(1-\bar{q} z)^{2}} \\
& +\alpha \lambda \int_{\mathbb{T} \backslash\{\bar{q}\}}\left(\ln \frac{1-\varkappa z}{1-\bar{q} z}+i(\operatorname{Im}\{\varkappa q\}) \frac{\bar{q} z}{1-\bar{q} z}\right) \frac{d \nu(\varkappa)}{1-\operatorname{Re}\{\varkappa q\}} .
\end{aligned}
$$


Очевидно, что полученное равенство для функции Кёнигса $F$ приводится к виду (7).

Обратно, пусть функция $F$ определяется формулой (7) с некоторыми $\beta \in \mathbb{R}$, $\lambda_{1}>0, \lambda_{2}, \lambda_{3} \geqslant 0$ и вероятностной мерой $\mu$ на $\mathbb{T} \backslash\{\bar{q}\}$. Тогда

$$
\begin{aligned}
F^{\prime}(z)= & i \beta \frac{\bar{q}}{(1-\bar{q} z)^{2}}+\lambda_{1} \frac{\bar{q}-\bar{a}}{(1-\bar{q} z)(1-\bar{a} z)}+\lambda_{2} \frac{\bar{q}(1+\bar{q} z)}{(1-\bar{q} z)^{3}} \\
& +\lambda_{3} \int_{\mathbb{T} \backslash\{\bar{q}\}}\left(\frac{\bar{q}-\varkappa}{(1-\bar{q} z)(1-\varkappa z)}+i(\operatorname{Im}\{\varkappa q\}) \frac{\bar{q}}{(1-\bar{q} z)^{2}}\right) \frac{d \mu(\varkappa)}{1-\operatorname{Re}\{\varkappa q\}} \\
= & \frac{p(z)}{q(1-\bar{q} z)^{2}},
\end{aligned}
$$

где

$$
p(z)=i \beta+\lambda_{1} \frac{(1-\bar{a} q)(1-\bar{q} z)}{1-\bar{a} z}+\lambda_{2} \frac{1+\bar{q} z}{1-\bar{q} z}+\lambda_{3} \int_{\mathbb{T} \backslash\{\bar{q}\}} \frac{1+\varkappa z}{1-\varkappa z} d \mu(\varkappa) .
$$

Замечая, что

$$
\frac{(1-\bar{a} q)(1-\bar{q} z)}{1-\bar{a} z}=(1-\operatorname{Re}\{\bar{a} q\}) \frac{1+\bar{a} z}{1-\bar{a} z}-i \operatorname{Im}\{\bar{a} q\}
$$

функцию $p$ представим в виде

$$
p(z)=\alpha\left(\frac{1+\bar{a} z}{1-\bar{a} z}+g(z)\right)
$$

где $\alpha=\lambda_{1}(1-\operatorname{Re}\{\bar{a} q\})>0$, а $g$ - голоморфная в $\mathbb{D}$ с неотрицательной вещественной частью функция. Следовательно,

$$
F^{\prime}(z)=\frac{\alpha}{q(1-\bar{q} z)^{2}}\left(\frac{1+\bar{a} z}{1-\bar{a} z}+g(z)\right),
$$

откуда видно, что $F$ является функцией Кёнигса однопараметрической полугруппы $t \mapsto f^{t}$ в $\mathfrak{P}[q ; a]$ с инфинитезимальной образующей

$$
v(z)=\frac{q(1-\bar{q} z)^{2}}{\alpha\left(\frac{1+\bar{a} z}{1-\bar{a} z}+g(z)\right)},
$$

и теорема 5 доказана.

\section{§ 7. Однопараметрические полугруппы с заданным множеством неподвижных точек}

Найти явный вид итераций аналитической функции удается крайне редко, хотя этот вопрос в приложениях часто является актуальным и тесно связан с динамикой описываемого процесса. Например, однопараметрические полугруппы вероятностных производящих функций являются аналитическим описанием однородных марковских ветвящихся процессов с непрерывным временем (см. [7]). Однопараметрические полугруппы инверсированных преобразований Коши вероятностных распределений связаны с описанием процессов с независимыми приращениями в некоммутативной вероятности (см. [18]). 
В теории композиционных операторов существенную роль играет наличие у функций однопараметрической полугруппы неподвижных точек (см. [19]). Кроме того, изучение неподвижных точек является центральной темой теории итераций. В связи с этим представляет интерес получение явных формул для функций Кёнигса, отвечающих однопараметрическим полугруппам в $\mathfrak{P}$ с заданными неподвижными точками. Этому вопросу и посвящен данный параграф.

Следующий результат можно рассматривать как дальнейшую детализацию формулы Берксона-Порты.

ПрЕДЛОЖЕНИЕ 1. Пустъ $q \in \overline{\mathbb{D}} u a_{1}, \ldots, a_{n}$ - попарно различные точки на единичной окружности $\mathbb{T}$. Тогда для любых положительных чисел $\alpha$, $\lambda_{1}, \ldots, \lambda_{n}$ и голоморфной в $\mathbb{D}$ функиии $p$ с неотрицательной вещественной частью равенство

$$
v(z)=\frac{(q-z)(1-\bar{q} z)}{\alpha\left(\sum_{k=1}^{n} \lambda_{k} \frac{1+\bar{a}_{k} z}{1-\bar{a}_{k} z}+p(z)\right)}
$$

определяет инфинитезимальную образующую однопараметрической полугрупnы $t \mapsto f^{t}$ в $\mathfrak{P}[q]$. При этом функиии $f^{t}, t>0$, оставляют неподвижсными точки $a_{1}, \ldots, a_{n}$ и имеют в этих точках конечные угловые производные.

ДокАЗАТЕЛьСтво. Заметим прежде всего, что поскольку

$$
\operatorname{Re}\left\{\sum_{k=1}^{n} \lambda_{k} \frac{1+\bar{a}_{k} z}{1-\bar{a}_{k} z}+p(z)\right\}>0
$$

при $z \in \mathbb{D}$, то функция $v$, определяемая по формуле (17), является инфинитезимальной образующей однопараметрической полугруппы $t \mapsto f^{t}$ в $\mathfrak{P}[q]$. Далее, для любого $k, k=1, \ldots, n$, функцию $v$ можно представить в виде

$$
v(z)=\frac{(q-z)(1-\bar{q} z)}{\alpha_{k}\left(\frac{1+\bar{a}_{k} z}{1-\bar{a}_{k} z}+g_{k}(z)\right)},
$$

где $\alpha_{k}=\alpha \lambda_{k}$ и

$$
g_{k}(z)=\sum_{j \neq k} \frac{\lambda_{j}}{\lambda_{k}} \frac{1+\bar{a}_{j} z}{1-\bar{a}_{j} z}+\frac{1}{\lambda_{k}} p(z) .
$$

Поскольку $\operatorname{Re} g_{k}(z)>0$ при $z \in \mathbb{D}$, то из теоремы 1 и леммы 2 следует, что $t \mapsto f^{t}$ является однопараметрической полугруппой в $\mathfrak{P}\left[q ; a_{k}\right]$. Это означает, что каждая точка $a_{k}, k=1, \ldots, n$, является неподвижной для функций $f^{t}$, $t>0$, и предложение доказано.

ПреДЛОЖЕНИЕ 2. Пусть $q \in \mathbb{D} u a_{1}, \ldots, a_{n}$ - попарно различные точки на единичной окружности $\mathbb{T}$. Тогда для любых положительных чисел $\lambda_{1}, \ldots, \lambda_{n}$ $u$ неотрицательного $\gamma$, удовлетворяющих условию $\lambda_{1}+\cdots+\lambda_{n}+\gamma=1$, $\sigma=e^{i \theta},-\pi / 2<\theta<\pi / 2$, и вероятностной меры $\mu$ на $\mathbb{T}$ равенство

$$
\begin{aligned}
F(z)=(z & -q)\left(\frac{1-\bar{q} z}{1-|q|^{2}}\right)^{\sigma^{2}} \prod_{k=1}^{n}\left(\frac{1-\bar{a}_{k} q}{1-\bar{a}_{k} z}\right)^{\lambda_{k}\left(1+\sigma^{2}\right)} \\
& \times \exp \left\{\gamma\left(1+\sigma^{2}\right) \int_{\mathbb{T}} \ln \frac{1-\varkappa q}{1-\varkappa z} d \mu(\varkappa)\right\},
\end{aligned}
$$


где под степенными функииями и логарифмом понимаются непрерывные ветви, которые принимают значения 1 и 0 соответственно при $z=q$, определяет функцию Кёнигса однопараметрической полугруппы $t \mapsto f^{t}$ в $\mathfrak{P}[q]$. При этом функиии $f^{t}, t>0$, оставляют неподвижными точки $a_{1}, \ldots, a_{n}$ и имеют в этих точках конечные угловые производные.

ДокАЗАТЕльство. Фиксируем произвольно $k, k=1, \ldots, n$, и заметим, что функцию $F$, определяемую равенством (18), можно также представить в виде

$$
\begin{aligned}
F(z)=(z & -q)\left(\frac{1-\bar{q} z}{1-|q|^{2}}\right)^{\sigma^{2}}\left(\frac{1-\bar{a}_{k} q}{1-\bar{a}_{k} z}\right)^{\lambda_{k}\left(1+\sigma^{2}\right)} \\
& \times \exp \left\{\left(1-\lambda_{k}\right)\left(1+\sigma^{2}\right) \int_{\mathbb{T}} \ln \frac{1-\varkappa q}{1-\varkappa z} d \mu_{k}(\varkappa)\right\},
\end{aligned}
$$

где вероятностная мера $\mu_{k}$ на $\mathbb{T}$ определяется как выпуклая комбинация

$$
\mu_{k}=\frac{\gamma}{1-\lambda_{k}} \mu+\sum_{j \neq k} \frac{\lambda_{j}}{1-\lambda_{k}} \delta_{\bar{a}_{j}},
$$

в которой под $\delta_{a}$ понимается мера Дирака на $\mathbb{T}$, единичная масса которой сосредоточена в точке $a$. Полученное представление функции $F$ вполне согласуется с видом (5). Следовательно, в силу теоремы 3 функции

$$
f^{t}(z)=F^{-1}\left(e^{-\sigma t} F(z)\right)
$$

где $t \geqslant 0$, корректно определены и образуют однопараметрическую полугруппу $t \mapsto f^{t}$ в $\mathfrak{P}\left[q ; a_{k}\right], k=1, \ldots, n$, т.е. функции $f^{t}, t>0$, оставляют неподвижными точки $a_{1}, \ldots, a_{n}$ и имеют в этих точках конечные угловые производные.

Аналогичный результат имеет место и в случае граничной точки ДанжуаВольфа.

ПРЕДЛОЖЕНИЕ 3. Пусть $q, a_{1}, \ldots, a_{n}$ - попарно различные точки на единичной окружности $\mathbb{T}$. Тогда для любых $\beta \in \mathbb{R}$, положительных $\lambda_{1}, \ldots, \lambda_{n}$, неотрицательных $\gamma_{1}, \gamma_{2}$ и вероятностной меры $\mu$ на $\mathbb{T} \backslash\{\bar{q}\}$ равенство

$$
\begin{aligned}
F(z)=i \beta & \frac{\bar{q} z}{1-\bar{q} z}+\sum_{k=1}^{n} \lambda_{k} \ln \frac{1-\bar{a}_{k} z}{1-\bar{q} z}+\gamma_{1} \frac{\bar{q} z}{(1-\bar{q} z)^{2}} \\
& +\gamma_{2} \int_{\mathbb{T} \backslash\{\bar{q}\}}\left(\ln \frac{1-\varkappa z}{1-\bar{q} z}+i(\operatorname{Im}\{\varkappa q\}) \frac{\bar{q} z}{1-\bar{q} z}\right) \frac{d \mu(\varkappa)}{1-\operatorname{Re}\{\varkappa q\}},
\end{aligned}
$$

где под логарифмами понимаются непрерывные ветви, обращающиеся в нуль при $z=0$, определяет функиию Кёнигса однопараметрической полугруппь $t \mapsto f^{t}$ в $\mathfrak{P}[q]$. При этом функиии $f^{t}, t>0$, оставляют неподвижными точки $a_{1}, \ldots, a_{n}$ и имеют в этих точках конечные угловые производные.

ДокАзАтЕльство. Пусть функция $F$ определяется равенством (19). Используя вычисления, проведенные в ходе доказательства теоремы 5 , получаем

$$
F^{\prime}(z)=\frac{p(z)}{q(1-\bar{q} z)^{2}},
$$


где

$$
\begin{aligned}
p(z)=i \beta & +\sum_{k=1}^{n} \lambda_{k} \frac{\left(1-\bar{a}_{k} q\right)(1-\bar{q} z)}{1-\bar{a}_{k} z}+\gamma_{1} \frac{1+\bar{q} z}{1-\bar{q} z}+\gamma_{2} \int_{\mathbb{T} \backslash\{\bar{q}\}} \frac{1+\varkappa z}{1-\varkappa z} d \mu(\varkappa) \\
= & i \beta+\sum_{k=1}^{n} \lambda_{k}\left(1-\operatorname{Re}\left\{\bar{a}_{k} q\right\}\right) \frac{1+\bar{a}_{k} z}{1-\bar{a}_{k} z}-i \sum_{k=1}^{n} \lambda_{k} \operatorname{Im}\left\{\bar{a}_{k} q\right\} \\
& +\gamma_{1} \frac{1+\bar{q} z}{1-\bar{q} z}+\gamma_{2} \int_{\mathbb{T} \backslash\{\bar{q}\}} \frac{1+\varkappa z}{1-\varkappa z} d \mu(\varkappa) .
\end{aligned}
$$

Отсюда видно, что $v(z)=1 / F^{\prime}(z)$ имеет вид (17), и доказываемое утверждение следует из предложения 1.

ЗАмЕчАниЕ 2. Класс функций Кёнигса, представленный в предложении 3 , содержит в себе следующее подмножество. Пусть $q, a_{1}, \ldots, a_{n}$ - попарно различные точки на единичной окружности $\mathbb{T}$. Тогда для любых $\beta \in \mathbb{R}$, положительных $\lambda_{1}, \ldots, \lambda_{n}$, неотрицательных $\gamma_{1}, \gamma_{2}$ и вероятностной меры $\mu$ на $\mathbb{T}$ равенство

$$
F(z)=i \beta \frac{\bar{q} z}{1-\bar{q} z}+\sum_{k=1}^{n} \lambda_{k} \ln \frac{1-\bar{a}_{k} z}{1-\bar{q} z}+\gamma_{1} \frac{\bar{q} z}{(1-\bar{q} z)^{2}}+\gamma_{2} \int_{\mathbb{T}} \ln \frac{1-\varkappa z}{1-\bar{q} z} d \mu(\varkappa),
$$

где под логарифмами понимаются непрерывные ветви, обращающиеся в нуль при $z=0$, определяет функцию Кёнигса однопараметрической полугруппы $t \mapsto f^{t}$ в $\mathfrak{P}[q]$. При этом функции $f^{t}, t>0$, оставляют неподвижными точки $a_{1}, \ldots, a_{n}$ и имеют в этих точках конечные угловые производные.

\section{Список литературы}

[1] E. Schröder, "Ueber iterirte Functionen", Math. Ann., 3:2 (1871), 296-322.

[2] G. Koenigs, "Recherches sur les intégrales de certaines équations fonctionnelles", Ann. Sci. École Norm. Sup. (3), 1 (1884), 3-41.

[3] E. Berkson, H. Porta, "Semigroups of analytic functions and composition operators", Michigan Math. J., 25:1 (1978), 101-115.

[4] В. В. Горяйнов, "Однопараметрические полугруппы аналитических функций и композиционный аналог безграничной делимости", Тр. ИПММ НАН Украины, 5 (2000), 44-57.

[5] K. Löwner, "Untersuchungen über schlichte konforme Abbildungen des Einheitskreises. I", Math. Ann., 89:1-2 (1923), 103-121.

[6] Ж. Валирон, Аналитические функиии, ГИТТЛ, М., 1957; пер. с англ.: G. Valiron, Fonctions analytiques, Presses Universitaires de France, Paris, 1954.

[7] Т. Харрис, Теория ветвящихся случайных прочессов, Мир, М., 1966; пер. с англ.: T. E. Harris, The theory of branching processes, Springer-Verlag, Berlin-GöttingenHeidelberg, 1963.

[8] M. D. Contreras, S. Díaz-Madrigal, Ch. Pommerenke, "Fixed points and boundary behaviour of the Koenigs function", Ann. Acad. Sci. Fenn. Math., 29:2 (2004), 471-488.

[9] В.В. Горяйнов, "Дробные итерации аналитических в единичном круге функций с заданными неподвижными точками", Матем. сб., 182:9 (1991), 1281-1299; англ. пер.: V. V. Goryajnov, "Fractional iterates of functions analytic in the unit disk, with given fixed points", Math. USSR-Sb., 74:1 (1993), 29-46. 
[10] L.V. Ahlfors, Conformal invariants. Topics in geometric function theory, McGraw-Hill, New York, 1973.

[11] Ch. Pommerenke, Boundary behaviour of conformal maps, Grundlehren Math. Wiss., 299, Springer-Verlag, Berlin, 1992.

[12] M. Kuczma, Functional equations in a single variable, Polish Scientific Publ., Warszawa, 1968.

[13] P. L. Duren, Univalent functions, Grundlehren Math. Wiss., 259, Springer-Verlag, New York-Berlin, 1983.

[14] Г. М. Голузин, Геометрическая теория функиий комплексного переменного, Наука, М., 1966; англ. пер.: G. M. Goluzin, Geometric theory of functions of a complex variable, Transl. Math. Monogr., 26, Amer. Math. Soc., Providence, RI, 1969.

[15] Ch. Pommerenke, "On the iteration of analytic functions in a halfplane. I", J. London Math. Soc. (2), 19:3 (1979), 439-447.

[16] I. N. Baker, Ch. Pommerenke, "On the iteration of analytic functions in a halfplane. II", J. London Math. Soc. (2), 20 (1979), 255-258.

[17] В. В. Горяйнов, “Функция Кёнигса и дробное итерирование вероятностных производящих функций”, Матем. сб., 193:7 (2002), 69-86; англ. пер.: V. V. Goryainov, "Koenigs function and fractional iterates of probability generating functions", $S b$. Math., 193:7 (2002), 1009-1025.

[18] U. Franz, N. Muraki, "Markov property of monotone Lévy processes", Infinite dimensional harmonic analysis. III (University of Tübingen, Tübingen, Germany, 2003), World Scientific, Hackensack, NJ, 2005, 37-57.

[19] J. H. Shapiro, Composition operators and classical function theory, Universitext Tracts Math., Springer-Verlag, New York, 1993.

\section{B. В. Горяйнов (V. V. Goryainov)}

Волжский гуманитарный институт

Волгоградского государственного университета,

г. Волжский, Волгоградская область

E-mail: goryainov_vv@hotmail.com

\section{O. С. Кудрявцева (O.S. Kudryavceva)}

Волжский гуманитарный институт

Волгоградского государственного университета,

г. Волжский, Волгоградская область

E-mail: kudryavceva@vgi.volsu.ru
Поступила в редакцию 09.03.2010 